TRANSACTIONS OF THE

AMERICAN MATHEMATICAL SOCIETY

Volume 365, Number 2, February 2013, Pages 591-621

S 0002-9947(2012)05774-6

Article electronically published on September 12, 2012

\title{
HYPERSURFACES OF CONSTANT HIGHER ORDER MEAN CURVATURE IN WARPED PRODUCTS
}

\author{
LUIS J. ALÍAS, DEBORA IMPERA, AND MARCO RIGOLI
}

\begin{abstract}
In this paper we characterize compact and complete hypersurfaces with some constant higher order mean curvature into warped product spaces. Our approach is based on the use of a new trace operator version of the OmoriYau maximum principle which seems to be interesting in its own.
\end{abstract}

\section{INTRODUCTION}

A classical result by Alexandrov [1] states that a compact hypersurface with constant mean curvature embedded in Euclidean space must be a round sphere. The original proof is based on a clever use of the maximum principle for elliptic partial differential equations. This method, now called the Alexandrov reflexion method, also works for hypersurfaces in ambient spaces having a sufficiently large number of isometric reflexions, for instance in the hyperbolic space.

To extend the above result to a larger class of Riemannian spaces it appears convenient to consider manifolds with a sufficiently large family of complete embedded constant mean curvature hypersurfaces. Such a family plays the role of the umbilical hypersurfaces in spaces of constant sectional curvature. In this setting, given an immersed hypersurface, the next step is to look for geometric assumptions that force the hypersurface to be one of the selected family. In the compact case, this was first done by Montiel [11, which considers as a natural class of ambient manifolds that of warped products $M^{n+1}=\mathbb{R} \times{ }_{\rho} \mathbb{P}^{n}$, where $\mathbb{P}^{n}$ is a complete $n$-dimensional Riemannian manifold and $\rho: \mathbb{R} \rightarrow \mathbb{R}_{+}$is a smooth warping function. Then each leaf $\mathbb{P}_{t}=\{t\} \times \mathbb{P}^{n}$ (called here a slice) of the foliation $t \in \mathbb{R} \mapsto \mathbb{P}_{t}$ of $M^{n+1}$ is a complete hypersurface with constant mean curvature. This approach was later considered in [4] where Alías and Dajczer generalized Montiel's results. Some of those generalizations hold even for complete, not necessarily compact, hypersurfaces.

The aim of the present paper is to extend the investigation to hypersurfaces with constant higher order mean curvatures, both in the compact and in the complete case. Our main analytical tools to reach the goal are provided by the Newton tensors $P_{k}$ of the hypersurface, their associated second order differential operators $L_{k}$, and further various combinations of them. We underline that in the complete

Received by the editors January 11, 2011.

2010 Mathematics Subject Classification. Primary 53C40, 53C42, 53A10.

The first author was partially supported by MICINN project MTM2009-10418 and Fundación Séneca project 04540/GERM/06, Spain. This research is a result of the activity developed within the framework of the Programme in Support of Excellence Groups of the Región de Murcia, Spain, by Fundación Séneca, Regional Agency for Science and Technology (Regional Plan for Science and Technology 2007-2010).

The third author was partially supported by MEC Grant SAB2010-0073. 
case, we tailor an appropriate version of the Omori-Yau maximum principle for very general operators to deal with the problems at hand.

The paper begins with Section 2, collecting a number of preliminary results and fixing notation. Section 3 is devoted to a proof of a generalized version of the OmoriYau maximum principle for a wide class of trace operators and to determining some geometrical assumptions guaranteeing the validity of the principle (see for instance Corollary 44). In Section 4, as a first application of our method and inspired by the mean curvature estimates given in $[3$, we derive higher order mean curvature estimates for complete immersed hypersurfaces. In Sections 5 and 6 we determine sufficient conditions for hypersurfaces with constant higher order mean curvatures contained in a slab to be a slice of the ambient space, extending previous results for the case of constant mean curvature hypersurfaces given in [4. Finally, in Section 7 we give a number of further results recovering this uniqueness property.

\section{Preliminaries}

Let $f: \Sigma^{n} \rightarrow M^{n+1}$ be a connected hypersurface isometrically immersed into the Riemannian manifold $M^{n+1}$. We let $A$ denote the second fundamental form of the immersion with respect to a (locally defined) normal vector field $N$. Its eigenvalues, $\kappa_{1}, \ldots, \kappa_{n}$, are the principal curvatures of the hypersurface (in the direction of $N$ ). Their elementary symmetric functions $S_{k}, k=0, \ldots, n, S_{0}=1$, define the $k$-mean curvatures of the immersion via the formula

$$
H_{k}=\left(\begin{array}{l}
n \\
k
\end{array}\right)^{-1} S_{k} .
$$

Thus $H_{1}=H$ is the mean curvature, $H_{n}$ is the Gauss-Kronecker curvature, and $H_{2}$ is, when the ambient space is Einstein, a multiple of the scalar curvature modulo a constant.

The Newton tensors associated to the immersion are inductively defined by

$$
P_{0}=I, \quad P_{k}=S_{k} I-A P_{k-1} .
$$

Note, for further use, that $\operatorname{Tr} P_{k}=(n-k) S_{k}$ and $\operatorname{Tr} A P_{k}=(k+1) S_{k+1}$. In the sequel we shall need to have the operators $P_{k}$ to be globally defined on $T \Sigma$. Obviously, the sign of the second fundamental form $A$ depends on the chosen local unit field $N$. However, when $k$ is even the sign of $S_{k}$ (and hence $H_{k}$ ) does not depend on the chosen $N$, which implies, by its very definition, that the operator $P_{k}$ is a globally defined tensor field on $T \Sigma$. On the other hand, when $k$ is odd in order to have $P_{k}$ globally defined, we need to assume that $\Sigma$ is two sided. Recall that a hypersurface $f: \Sigma^{n} \rightarrow M^{n+1}$ is called two sided if its normal bundle is trivial, i.e. there exists a globally defined unit normal vector field $N$. For instance, every hypersurface with never vanishing mean curvature is trivially two sided. When the hypersurface is two sided, a choice of $N$ on $\Sigma$ makes the second fundamental form $A$ and its associated Newton tensors $P_{k}$ globally defined tensor fields on $T \Sigma$.

Let $\nabla$ stand for the Levi-Civita connection of $\Sigma$. For a given function $u \in C^{2}(\Sigma)$, we denote by hess $u: T \Sigma \rightarrow T \Sigma$ the symmetric operator given by hess $u(X)=$ $\nabla_{X} \nabla u$ for every $X \in T \Sigma$, and by Hess $u: T \Sigma \times T \Sigma \rightarrow C^{\infty}(\Sigma)$ the metrically equivalent bilinear form given by

$$
\text { Hess } u(X, Y)=\langle\operatorname{hess} u(X), Y\rangle \text {. }
$$


Associated to each globally defined Newton tensor $P_{k}: T \Sigma \rightarrow T \Sigma$, we may consider the second order differential operator $L_{k}: \mathcal{C}^{\infty}(\Sigma) \rightarrow \mathcal{C}^{\infty}(\Sigma)$ given by $L_{k}=$ $\operatorname{Tr}\left(P_{k} \circ\right.$ hess $)$. In particular, $L_{0}$ is the Laplace-Beltrami operator $\Delta$. Observe that

$$
L_{k}(u)=\operatorname{div}\left(P_{k} \nabla u\right)-\left\langle\operatorname{div} P_{k}, \nabla u\right\rangle,
$$

where $\operatorname{div} P_{k}=\operatorname{Tr} \nabla P_{k}$. This implies that $L_{k}$ is elliptic if and only if $P_{k}$ is positive definite, and in this case the maximum principle holds for $L_{k}$. See for instance Theorem 3.1 in [10].

Note that the ellipticity of the operator $L_{1}$ is guaranteed by the assumption $H_{2}>0$. Indeed, if this happens the mean curvature does not vanish on $\Sigma$, because of the basic inequality $H_{1}^{2} \geq H_{2}$. Therefore, the immersion is two sided and we can choose the normal unit vector $N$ on $\Sigma$ so that $H_{1}>0$. Furthermore

$$
n^{2} H_{1}^{2}=\sum_{j=1}^{n} \kappa_{j}^{2}+n(n-1) H_{2}>\kappa_{i}^{2}
$$

for every $i=1, \ldots, n$, and then the eigenvalues of $P_{1}$ satisfy $\mu_{1, i}=n H_{1}-\kappa_{i}>0$ for every $i$ (see, for instance, Lemma 3.10 in [8]). This shows ellipticity of $L_{1}$. Regarding the ellipticity of $L_{j}$ when $j \geq 2$, we will assume that there exists an elliptic point in $\Sigma$, that is, a point $p \in \Sigma$ at which the second fundamental form $A$ is positive definite with respect to an appropriate orientation. The existence of an elliptic point implies that $H_{k}$ is positive at that point, and applying Gårding inequalities [9], we have

$$
H_{1} \geq H_{2}^{1 / 2} \geq \cdots \geq H_{k-1}^{1 /(k-1)} \geq H_{k}^{1 / k}>0,
$$

with equality at any stage only for an umbilical point. Therefore, in case $H_{k}$ is constant, the immersion is two sided and $H_{1}>0$ for the chosen orientation. Moreover, in this case, for every $1 \leq j \leq k-1$, the operators $L_{j}$ are elliptic or, equivalently, the operators $P_{j}$ are positive definite (for a proof of this fact, see [7. Proposition 3.2]). Observe that the existence of an elliptic point is not guaranteed, in general, even in the compact case. For instance, it is clear that totally geodesic spheres and Clifford tori in $\mathbb{S}^{n+1}$ are examples of compact isoparametric hypersurfaces without elliptic points. On the contrary, it is not difficult to see that every compact hypersurface in an open hemisphere has elliptic points (see for instance the proof of Theorem 11.1 in [5]).

In what follows, we consider the case when the ambient space is a warped product $M^{n+1}=I \times{ }_{\rho} \mathbb{P}^{n}$, where $I \subseteq \mathbb{R}$ is an open interval, $\mathbb{P}^{n}$ is a complete $n$-dimensional Riemannian manifold, and $\rho: I \rightarrow \mathbb{R}_{+}$is a smooth function. The product manifold $I \times \mathbb{P}^{n}$ is endowed with the Riemannian metric

$$
\langle,\rangle=\pi_{I}^{*}\left(\mathrm{~d} t^{2}\right)+\rho^{2}\left(\pi_{I}\right) \pi_{\mathbb{P}}^{*}\left(\langle,\rangle_{\mathbb{P}}\right) .
$$

Here $\pi_{I}$ and $\pi_{\mathbb{P}}$ denote the projections onto the corresponding factor and $\langle,\rangle_{\mathbb{P}}$ is the Riemannian metric on $\mathbb{P}^{n}$. In particular, $M^{n+1}=I \times{ }_{\rho} \mathbb{P}^{n}$ is complete if and only if $I=\mathbb{R}$. We also observe that each leaf $\mathbb{P}_{t}=\{t\} \times \mathbb{P}^{n}$ of the foliation $t \rightarrow \mathbb{P}_{t}$ of $M^{n+1}$ is a complete totally umbilical hypersurface with constant $k$-mean curvature

$$
\mathcal{H}_{k}(t)=\left(\frac{\rho^{\prime}(t)}{\rho(t)}\right)^{k}, \quad 0 \leq k \leq n,
$$

with respect to $-\partial / \partial t$. 
Let $f: \Sigma^{n} \rightarrow M^{n+1}=I \times{ }_{\rho} \mathbb{P}^{n}$ be an isometrically immersed hypersurface. We define the height function $h \in C^{\infty}(\Sigma)$ by setting $h=\pi_{I} \circ f$. In this context and following the terminology introduced in [3], we will say that the hypersurface is contained in a slab if $f(\Sigma)$ lies between two leaves $\mathbb{P}_{t_{1}}, \mathbb{P}_{t_{2}}$ with $t_{1}<t_{2}$ of the foliation.

We observe that results similar to those of the present paper hold for spacelike hypersurfaces in a generalized Robertson-Walker spacetime. These will appear in our paper $[6]$.

\section{The generalized OMOrI-Yau MaXimum PRINCIPle FOR TRACE OPERATORS}

Let $\Sigma^{n}$ be an $n$-dimensional Riemannian manifold. Following the terminology introduced in [14, the Omori-Yau maximum principle is said to hold on $\Sigma$ for the Laplace operator if, for any smooth function $u \in \mathcal{C}^{2}(\Sigma)$ with $u^{*}=\sup _{\Sigma} u<+\infty$, there exists a sequence of points $\left\{p_{j}\right\}_{j \in \mathbb{N}}$ in $\Sigma$ with the properties

$$
\text { (i) } u\left(p_{j}\right)>u^{*}-\frac{1}{j} \text {, (ii) }\left\|\nabla u\left(p_{j}\right)\right\|<\frac{1}{j} \text {, and (iii) } \Delta u\left(p_{j}\right)<\frac{1}{j} \text {. }
$$

Equivalently, for any $u \in \mathcal{C}^{2}(\Sigma)$ with $u_{*}=\inf _{\Sigma} u>-\infty$ there exists a sequence $\left\{p_{j}\right\}_{j \in \mathbb{N}}$ in $\Sigma$ satisfying

$$
\text { (i) } u\left(p_{j}\right)<u_{*}+\frac{1}{j} \text {, (ii) }\left\|\nabla u\left(p_{j}\right)\right\|<\frac{1}{j} \text {, and (iii) } \Delta u\left(p_{j}\right)>-\frac{1}{j} \text {. }
$$

In this sense, the classical result given by Omori [12] and Yau [15] states that the Omori-Yau maximum principle holds on every complete Riemannian manifold with Ricci curvature bounded from below. More generally, as shown by Pigola, Rigoli and Setti [14, Example 1.13], a sufficiently controlled decay of the radial Ricci curvature of the form

$$
\operatorname{Ric}_{\Sigma}(\nabla r, \nabla r) \geq-C^{2} G(r)
$$

where $r$ is the distance function on $\Sigma$ to a fixed point, $C$ a positive constant, and $G:[0,+\infty) \rightarrow \mathbb{R}$ is a smooth function satisfying

$$
\begin{gathered}
\text { (i) } G(0)>0, \text { (ii) } G^{\prime}(t) \geq 0 \text {, (iii) } \int_{0}^{+\infty} 1 / \sqrt{G(t)}=+\infty \text {, and } \\
\text { (iv) } \limsup _{t \rightarrow+\infty} t G(\sqrt{t}) / G(t)<+\infty,
\end{gathered}
$$

suffices to imply the validity of the Omori-Yau maximum principle.

On the other hand, as observed again in [14], the validity of Omori-Yau maximum principle on $\Sigma^{n}$ does not depend on curvature bounds as much as one would expect. For instance, the Omori-Yau maximum principle holds on every Riemannian manifold admitting a non-negative $C^{2}$ function $\gamma$ satisfying the following requirements: (i) $\gamma(p) \rightarrow+\infty$ as $p \rightarrow \infty$; (ii) there exists $A>0$ such that $\|\nabla \gamma\| \leq A \sqrt{\gamma}$ off a compact set; and (iii) there exists $B>0$ such that $\Delta \gamma \leq B \sqrt{\gamma} \sqrt{G(\sqrt{\gamma})}$ off a compact set, where $G$ is as above (see [14, Theorem 1.9]).

For the proof of our main results in this paper, we will use the following generalization of [14, Theorem 1.9] for trace type differential operators which includes the operators $L_{k}$. 
Theorem 1. Let $(\Sigma,\langle\rangle$,$) be a Riemannian manifold, and let L=\operatorname{Tr}(P \circ$ hess $)$ be a semi-elliptic operator, where $P: T \Sigma \rightarrow T \Sigma$ is a positive semi-definite symmetric tensor satisfying $\sup _{\Sigma} \operatorname{Tr} P<+\infty$. Assume the existence of a non-negative $C^{2}$ function $\gamma$ with the properties

$$
\begin{aligned}
\gamma(p) \rightarrow+\infty & \text { as } p \rightarrow \infty, \\
\exists A>0 & \text { such that }\|\nabla \gamma\| \leq A \gamma^{\frac{1}{2}} \quad \text { off a compact set, } \\
\exists B>0 & \text { such that } L \gamma \leq B \gamma^{\frac{1}{2}} G\left(\gamma^{\frac{1}{2}}\right)^{\frac{1}{2}} \quad \text { off a compact set, }
\end{aligned}
$$

where $G$ is a smooth function on $[0,+\infty)$ such that

$$
\begin{array}{ll}
\text { (i) } G(0)>0 ; & \text { (ii) } G^{\prime}(t) \geq 0 \quad \text { on }[0,+\infty) ; \\
\text { (iii) } G(t)^{-\frac{1}{2}} \notin L^{1}(+\infty) ; & \text { (iv) } \limsup _{t \rightarrow \infty} \frac{t G\left(t^{\frac{1}{2}}\right)}{G(t)}<+\infty .
\end{array}
$$

Then, given any function $u \in C^{2}(\Sigma)$ with $u^{*}=\sup _{\Sigma} u<+\infty$, there exists a sequence $\left\{p_{j}\right\}_{j \in \mathbb{N}} \subset \Sigma$ with the properties

$$
\text { (i) } u\left(p_{j}\right)>u^{*}-\frac{1}{j} \text {, (ii) }\left\|\nabla u\left(p_{j}\right)\right\|<\frac{1}{j} \text {, (iii) } L u\left(p_{j}\right)<\frac{1}{j}, \forall j \in \mathbb{N} \text {. }
$$

Proof. Define the function

$$
\varphi(t)=e^{\int_{0}^{t} G(s)^{-\frac{1}{2}}} \mathrm{~d} s .
$$

Note that $\varphi(t)$ is a well defined, smooth, positive function such that $\varphi(t) \rightarrow+\infty$ as $t \rightarrow+\infty$. Moreover

$$
\varphi^{\prime}(t)=G(t)^{-\frac{1}{2}} \varphi(t) \quad \text { and } \quad \varphi^{\prime \prime}(t)=\left(G(t)^{-1}-2 G(t)^{-\frac{3}{2}} G^{\prime}(t)\right) \varphi(t),
$$

and therefore

$$
\left(\frac{\varphi^{\prime}(t)}{\varphi(t)}\right)^{2}-\frac{\varphi^{\prime \prime}(t)}{\varphi(t)}=2 G(t)^{-\frac{3}{2}} G^{\prime}(t) \geq 0 .
$$

Then, using assumption (5), (iv) we get

$$
\frac{\varphi^{\prime}(t)}{\varphi(t)} \leq c\left(t G\left(t^{\frac{1}{2}}\right)\right)^{-\frac{1}{2}}
$$

for some constant $c>0$.

Fix a point $p_{0} \in \Sigma$ and, for a fixed $j \in \mathbb{N}$ define

$$
f_{j}(p)=\frac{u(p)-u\left(p_{0}\right)+1}{\varphi(\gamma(p))^{\frac{1}{j}}} .
$$

Then $f_{j}\left(p_{0}\right)=1 / \varphi\left(\gamma\left(p_{0}\right)\right)^{1 / j}>0$. Moreover, since $u^{*}<+\infty$ and $\varphi(\gamma(p)) \rightarrow+\infty$ as $p \rightarrow \infty$, we have $\lim \sup _{p \rightarrow \infty} f_{j}(p) \leq 0$. Thus, $f_{j}$ attains a positive absolute maximum at $p_{j} \in \Sigma$. Iterating this procedure, we produce a sequence $\left\{p_{j}\right\}_{j \in \mathbb{N}} \subset \Sigma$. The proof of ([6), (i) and ([6), (ii) is the same as in [14], so we only prove ([6),(iii). Proceeding as in Theorem 1.9 of [14], up to passing to a subsequence, we have $\lim _{j \rightarrow+\infty} u\left(p_{j}\right)=u^{*}$. If $\left\{p_{j}\right\}$ is contained in a compact set, then $p_{j} \rightarrow \bar{p} \in \Sigma$ as $j \rightarrow+\infty$ and $u$ attains its absolute maximum. Hence we have

$$
u(\bar{p})=u^{*}, \quad\|\nabla u(\bar{p})\|=0, \quad \operatorname{Hess} u(\bar{p}) \leq 0 .
$$

In particular, since $P$ is positive semi-definite, it holds that $L u(\bar{p}) \leq 0$. Hence the sequence $p_{j}=\bar{p}$, for each $j$, satisfies all the requirements. Now consider the case 
when $\left\{p_{j}\right\}$ diverges off a compact set, so that, according to (2), $\gamma\left(p_{j}\right) \rightarrow+\infty$. Since $f_{j}$ attains a positive maximum at $p_{j}$, we have
(i) $\left(\nabla \log f_{j}\right)\left(p_{j}\right)=0$,
(ii) $\operatorname{Hess} \log f_{j}\left(p_{j}\right) \leq 0$.

A simple computation then gives

$$
\begin{aligned}
\operatorname{Hess} u\left(p_{j}\right)(v, v) \leq & \frac{1}{j}\left(u\left(p_{j}\right)-u\left(p_{0}\right)+1\right)\left\{\frac{\varphi^{\prime}\left(\gamma\left(p_{j}\right)\right)}{\varphi\left(\gamma\left(p_{j}\right)\right)} \operatorname{Hess} \gamma\left(p_{j}\right)(v, v)\right. \\
& \left.+\left[\left(\frac{1}{j}-1\right)\left(\frac{\varphi^{\prime}\left(\gamma\left(p_{j}\right)\right)}{\varphi\left(\gamma\left(p_{j}\right)\right)}\right)^{2}+\frac{\varphi^{\prime \prime}\left(\gamma\left(p_{j}\right)\right)}{\varphi\left(\gamma\left(p_{j}\right)\right)}\right]\left\langle\nabla \gamma\left(p_{j}\right), v\right\rangle^{2}\right\} \\
\leq & \frac{1}{j}\left(u\left(p_{j}\right)-u\left(p_{0}\right)+1\right)\left\{\frac{\varphi^{\prime}\left(\gamma\left(p_{j}\right)\right)}{\varphi\left(\gamma\left(p_{j}\right)\right)} \operatorname{Hess} \gamma\left(p_{j}\right)(v, v)\right. \\
& \left.+\frac{1}{j}\left(\frac{\varphi^{\prime}\left(\gamma\left(p_{j}\right)\right)}{\varphi\left(\gamma\left(p_{j}\right)\right)}\right)^{2}\left\langle\nabla \gamma\left(p_{j}\right), v\right\rangle^{2}\right\},
\end{aligned}
$$

for every $v \in T_{p_{j}} \Sigma$, where we have used (7). Let $\left\{e_{1}, \ldots, e_{n}\right\} \subset T_{p_{j}} \Sigma$ be an orthonormal basis of eigenvectors of $P\left(p_{j}\right)$ corresponding to the eigenvalues $\mu_{i}\left(p_{j}\right)=$ $\left\langle P\left(p_{j}\right) e_{i}, e_{i}\right\rangle \geq 0$. Then, for every $1 \leq i \leq n$, we have

$$
\begin{aligned}
\left\langle P \operatorname{hess} u\left(p_{j}\right) e_{i}, e_{i}\right\rangle= & \mu_{i}\left(p_{j}\right) \operatorname{Hess} u\left(p_{j}\right)\left(e_{i}, e_{i}\right) \\
\leq & \frac{1}{j}\left(u\left(p_{j}\right)-u\left(p_{0}\right)+1\right)\left\{\frac{\varphi^{\prime}\left(\gamma\left(p_{j}\right)\right)}{\varphi\left(\gamma\left(p_{j}\right)\right)}\left\langle P \operatorname{hess} \gamma\left(p_{j}\right) e_{i}, e_{i}\right\rangle\right. \\
& \left.+\frac{1}{j}\left(\frac{\varphi^{\prime}\left(\gamma\left(p_{j}\right)\right)}{\varphi\left(\gamma\left(p_{j}\right)\right)}\right)^{2} \mu_{i}\left(p_{j}\right)\left\langle\nabla \gamma\left(p_{j}\right), e_{i}\right\rangle^{2}\right\} .
\end{aligned}
$$

Taking traces here and using the fact that

$$
\langle P \nabla \gamma, \nabla \gamma\rangle=\sum_{i=1}^{n} \mu_{i}\left\langle\nabla \gamma, e_{i}\right\rangle^{2} \leq \operatorname{Tr} P\|\nabla \gamma\|^{2},
$$

we obtain that

$$
\begin{aligned}
L u\left(p_{j}\right) \leq & \frac{1}{j}\left(u\left(p_{j}\right)-u\left(p_{0}\right)+1\right)\left\{\frac{\varphi^{\prime}\left(\gamma\left(p_{j}\right)\right)}{\varphi\left(\gamma\left(p_{j}\right)\right)} L \gamma\left(p_{j}\right)\right. \\
& \left.+\frac{1}{j}\left(\frac{\varphi^{\prime}\left(\gamma\left(p_{j}\right)\right)}{\varphi\left(\gamma\left(p_{j}\right)\right)}\right)^{2}\left\langle P \nabla \gamma\left(p_{j}\right), \nabla \gamma\left(p_{j}\right)\right\rangle\right\} \\
\leq & \frac{1}{j}\left(u\left(p_{j}\right)-u\left(p_{0}\right)+1\right)\left\{\frac{\varphi^{\prime}\left(\gamma\left(p_{j}\right)\right)}{\varphi\left(\gamma\left(p_{j}\right)\right)} L \gamma\left(p_{j}\right)\right. \\
& \left.+\frac{1}{j}\left(\frac{\varphi^{\prime}\left(\gamma\left(p_{j}\right)\right)}{\varphi\left(\gamma\left(p_{j}\right)\right)}\right)^{2} \operatorname{Tr} P\left\|\nabla \gamma\left(p_{j}\right)\right\|^{2}\right\} .
\end{aligned}
$$

Since (3) and (4) hold outside a compact set, they hold at $p_{j}$ for $j$ sufficiently large. Then, using (8),

$$
\begin{aligned}
L u\left(p_{j}\right) & \leq \frac{1}{j}\left(u\left(p_{j}\right)-u\left(p_{0}\right)+1\right)\left\{B c+\frac{1}{j} c^{2} A^{2} C G\left(\gamma\left(p_{j}\right)^{\frac{1}{2}}\right)^{-1}\right\} \\
& \leq C \frac{u^{*}-u\left(p_{0}\right)+1}{j}
\end{aligned}
$$

for some constant $C>0$. Since the right-hand side tends to zero as $j \rightarrow+\infty$, this proves condition (iii) in (6) . 
Following the terminology introduced in [14, we introduce the next

Definition 2. Let $\Sigma$ be a Riemannian manifold, and let $L$ be an operator as in Theorem 1. The Omori-Yau maximum principle is said to hold on $\Sigma$ for the operator $L$ if, for any function $u \in C^{2}(\Sigma)$ with $u^{*}=\sup _{\Sigma} u<+\infty$, there exists a sequence $\left\{p_{j}\right\}_{j \in \mathbb{N}} \subset \Sigma$ with the properties
(i) $u\left(p_{j}\right)>u^{*}-\frac{1}{j}$,
(ii) $\left\|\nabla u\left(p_{j}\right)\right\|<\frac{1}{j}$
(iii) $L u\left(p_{j}\right)<\frac{1}{j}$

for every $j \in \mathbb{N}$. Equivalently, for any function $u \in C^{2}(\Sigma)$ with $u_{*}=\inf _{\Sigma} u>-\infty$, there exists a sequence $\left\{p_{j}\right\}_{j \in \mathbb{N}} \subset \Sigma$ with the properties

$$
\text { (i) } u\left(p_{j}\right)<u_{*}+\frac{1}{j} \text {, (ii) }\left\|\nabla u\left(p_{j}\right)\right\|<\frac{1}{j} \text {, (iii) } L u\left(p_{j}\right)>-\frac{1}{j}
$$

for every $j \in \mathbb{N}$.

The function theoretic approach to the generalized Omori-Yau maximum principle given in Theorem 1 allows us to apply it in different situations, where the choices of the functions $\gamma$ and $G$ are suggested by the geometric setting. The next are two significant and useful examples of an intrinsic and extrinsic nature, respectively.

Let $(\Sigma,\langle\rangle$,$) be a complete, non-compact Riemannian manifold, and let o \in \Sigma$ be a fixed reference point. Denote with $r(p)$ the distance function from $o$ and set $\gamma(p)=r(p)^{2}$. Then $\gamma$ satisfies assumptions (2) and (3) of Theorem 1, Furthermore, $\gamma$ is smooth within the cut locus of $o$. Assume that the radial sectional curvature of $\Sigma$, that is, the sectional curvature of the 2-planes containing $\nabla r$, satisfies

$$
K_{\Sigma}^{\mathrm{rad}} \geq-G(r)
$$

where $G$ is a smooth function on $[0,+\infty)$ even at the origin, i.e. $G^{(2 k+1)}(0)=0$ for each $k=0,1, \ldots$, and satisfying conditions (i)-(iv) listed in (5). Then assumption (41) is satisfied.

Indeed, assuming that (9) holds, by the Hessian comparison theorem within the cut locus of $o$, one has

$$
\operatorname{Hess} r(p)(v, v) \leq \frac{\phi^{\prime}(r(p))}{\phi(r(p))}\left(\|v\|^{2}-\langle\nabla r(p), v\rangle^{2}\right)
$$

for every $v \in T_{p} \Sigma$, where $\phi(t)$ is the (positive) solution of the initial value problem

$$
\left\{\begin{array}{l}
\phi^{\prime \prime}-G \phi=0 \\
\phi(0)=0, \phi^{\prime}(0)=1
\end{array}\right.
$$

Now let

$$
\psi(t)=\frac{1}{\sqrt{G(0)}}\left(e^{\int_{0}^{t} \sqrt{G(s)} d s}-1\right) .
$$

Then $\psi(0)=0, \psi^{\prime}(0)=1$ and

$$
\psi^{\prime \prime}(t)-G(t) \psi(t)=\frac{1}{\sqrt{G(0)}}\left(G(t)+\frac{G^{\prime}(t)}{2 \sqrt{G(t)}} e^{\int_{0}^{t} \sqrt{G(s)} d s}\right) \geq 0 .
$$

Hence, by the Sturm comparison theorem

$$
\frac{\phi^{\prime}(t)}{\phi(t)} \leq \frac{\psi^{\prime}(t)}{\psi(t)}=\sqrt{G(t)} \frac{e^{\int_{0}^{t} \sqrt{G(s)} d s}}{e^{\int_{0}^{t} \sqrt{G(s)} d s}-1} \leq c \sqrt{G(t)},
$$


where the last inequality holds for a constant $c>0$ and $t$ sufficiently large. Therefore, if $r$ is sufficiently large

$$
\text { Hess } r \leq c \sqrt{G(r)}(\langle,\rangle-d r \otimes d r) .
$$

Since Hess $\gamma=2 r$ Hess $r+2 d r \otimes d r$, we obtain from here that

$$
\text { Hess } \gamma \leq c \sqrt{\gamma G(\sqrt{\gamma})}\langle,\rangle
$$

for a constant $c$ and $\gamma$ sufficiently large. Then, using the fact that $P$ is positive semi-definite

$$
L \gamma \leq c \operatorname{Tr} P \sqrt{\gamma G(\sqrt{\gamma})}
$$

for a constant $c$ and $\gamma$ sufficiently large. We have thus proved the following:

Corollary 3. Let $(\Sigma,\langle\rangle$,$) be a complete, non-compact Riemannian manifold whose$ radial sectional curvature satisfies condition (9). Then the Omori-Yau maximum principle holds on $\Sigma$ for any semi-elliptic operator $L=\operatorname{Tr}(P \circ$ hess $)$ with $\sup _{\Sigma} \operatorname{Tr} P<$ $+\infty$.

On the other hand, the following example, which is of an extrinsic nature, will be useful in the sequel for the case of properly immersed hypersurfaces. Consider $\mathbb{P}^{n}$ a complete, non-compact, Riemannian manifold, let $o \in \mathbb{P}^{n}$ be a reference point, and denote by $\hat{r}$ the distance function from $o$. We will assume that the radial sectional curvature of $\mathbb{P}^{n}$ satisfies the condition

$$
K_{\mathbb{P}}^{\mathrm{rad}} \geq-G(\hat{r}),
$$

where $G$ is a smooth function on $[0,+\infty)$ even at the origin, i.e. $G^{(2 k+1)}(0)=0$ for each $k=0,1, \ldots$, and satisfying conditions (i)-(iv) listed in (5). Let $f: \Sigma^{n} \rightarrow$ $M^{n+1}=I \times{ }_{\rho} \mathbb{P}^{n}$ be a hypersurface. Observe that if $\Sigma$ is compact, then every immersion $f: \Sigma^{n} \rightarrow I \times{ }_{\rho} \mathbb{P}^{n}$ is proper and contained in a slab, and the Omori-Yau maximum principle trivially holds on $\Sigma$ for any semi-elliptic operator. Assume then that $\Sigma$ is non-compact, and let $f: \Sigma^{n} \rightarrow M^{n+1}=I \times{ }_{\rho} \mathbb{P}^{n}$ be a properly immersed hypersurface which is contained in a slab, that is, $f(\Sigma) \subset\left[t_{1}, t_{2}\right] \times \mathbb{P}^{n}$.

Let $\hat{\gamma}: \mathbb{P}^{n} \rightarrow \mathbb{R}$ be the function given by $\hat{\gamma}(x)=\hat{r}(x)^{2}$ for every $x \in \mathbb{P}^{n}$, and set $\gamma: \Sigma \rightarrow \mathbb{R}$ for the associated function, defined as

$$
\gamma(p)=\tilde{\gamma}(f(p))=\hat{\gamma}(x(p))=\hat{r}(x(p))^{2}
$$

for every $p \in \Sigma$, where $\tilde{\gamma}(t, x)=\hat{\gamma}(x)$ and $f(p)=(h(p), x(p))$. Since $f$ is proper, if $p \rightarrow \infty$ in $\Sigma$, then $f(p) \rightarrow \infty$ in $M^{n+1}=I \times{ }_{\rho} \mathbb{P}^{n}$, but being $f$ contained in a slab, this means that $x(p) \rightarrow \infty$ in $\mathbb{P}^{n}$. It follows that $\gamma(p)=\hat{r}(x(p))^{2} \rightarrow+\infty$ as $p \rightarrow \infty$ in $\Sigma$, and $\gamma$ satisfies condition (2) in Theorem 1.

Let us denote by $\tilde{\nabla}, \hat{\nabla}$, and $\nabla$ the Levi-Civita connection (and the gradient operators) in $M^{n+1}, \mathbb{P}^{n}$, and $\Sigma^{n}$, respectively. Since $\gamma=\tilde{\gamma} \circ f$, along the immersion $f$ we have

$$
\tilde{\nabla} \tilde{\gamma}=\nabla \gamma+\langle\tilde{\nabla} \tilde{\gamma}, N\rangle N
$$

where $N$ is a (local) smooth unit normal field along $f$. On the other hand, from $\tilde{\gamma}(t, x)=\hat{\gamma}(x)$ we have

$$
\langle\tilde{\nabla} \tilde{\gamma}, T\rangle=0
$$

where, as usual, $T$ stands for the lift of $\partial_{t}$ to the product $I \times \mathbb{P}^{n}$, and

$$
\langle\tilde{\nabla} \tilde{\gamma}, V\rangle=\langle\hat{\nabla} \hat{\gamma}, V\rangle_{\mathbb{P}}
$$


for every $V$, where $V$ denotes the lift of a vector field $V \in T P$ to $I \times \mathbb{P}^{n}$. Since

$$
\langle\tilde{\nabla} \tilde{\gamma}, V\rangle=\rho^{2}\langle\tilde{\nabla} \tilde{\gamma}, V\rangle_{\mathbb{P}}
$$

we conclude from here that

$$
\tilde{\nabla} \tilde{\gamma}=\frac{1}{\rho^{2}} \hat{\nabla} \hat{\gamma}=\frac{2 \hat{r}}{\rho^{2}} \hat{\nabla} \hat{r}
$$

Therefore, since $\|\hat{\nabla} \hat{r}\|=\rho\|\hat{\nabla} \hat{r}\|_{\mathbb{P}}=\rho$ and $\rho(h) \geq \min _{\left[t_{1}, t_{2}\right]} \rho(t)>0$, along the immersion we have

$$
\|\nabla \gamma\| \leq\|\tilde{\nabla} \tilde{\gamma}\|=\frac{2 \sqrt{\gamma}}{\rho(h)} \leq c \sqrt{\gamma}
$$

for a positive constant $c$. Thus, $\gamma$ also satisfies condition (3) in Theorem 11. In particular, $\Sigma$ is complete (see [14] pag 10).

Next, we will see that, under appropriate extrinsic restrictions, condition (4) in Theorem 1 is also satisfied. From (14) it follows that

$$
\operatorname{Hess} \gamma(X, X)=\operatorname{Hess} \tilde{\gamma}(X, X)+\langle\tilde{\nabla} \tilde{\gamma}, N\rangle\langle A X, X\rangle
$$

for every tangent vector field $X \in T \Sigma$. From (15)

$$
\tilde{\nabla}_{T} \tilde{\nabla} \tilde{\gamma}=-\frac{\rho^{\prime}}{\rho^{3}} \hat{\nabla} \hat{\gamma}=-\mathcal{H} \tilde{\nabla} \tilde{\gamma}
$$

where $\mathcal{H}(t)=\rho^{\prime}(t) / \rho(t)$. In particular, Hess $\tilde{\gamma}(T, T)=0$. Then, writing $X=$ $X^{*}+\langle X, T\rangle T$, where $X^{*}=\pi_{\mathbb{P} *} X$, we have

$$
\operatorname{Hess} \tilde{\gamma}(X, X)=\operatorname{Hess} \tilde{\gamma}\left(X^{*}, X^{*}\right)+2\langle X, T\rangle \operatorname{Hess} \tilde{\gamma}\left(X^{*}, T\right) \text {. }
$$

From (17) we have that

$$
\operatorname{Hess} \tilde{\gamma}\left(X^{*}, T\right)=-\mathcal{H}(h)\langle\tilde{\nabla} \tilde{\gamma}, X\rangle=-\mathcal{H}(h)\langle\nabla \gamma, X\rangle .
$$

On the other hand, using

$$
\tilde{\nabla}_{X *} \tilde{\nabla} \tilde{\gamma}=\frac{1}{\rho^{2}} \hat{\nabla}_{X^{*}} \hat{\nabla} \hat{\gamma}-\frac{\rho^{\prime}}{\rho^{3}}\left\langle\hat{\nabla} \hat{\gamma}, X^{*}\right\rangle T
$$

we also have

$$
\operatorname{Hess} \tilde{\gamma}\left(X^{*}, X^{*}\right)=\frac{1}{\rho^{2}}\left\langle\hat{\nabla}_{X^{*}} \hat{\nabla} \hat{\gamma}, X^{*}\right\rangle=\left\langle\hat{\nabla}_{X^{*}} \hat{\nabla} \hat{\gamma}, X^{*}\right\rangle_{\mathbb{P}}=\operatorname{Hess} \hat{\gamma}\left(X^{*}, X^{*}\right) .
$$

Summing up,

$$
\begin{aligned}
\operatorname{Hess} \gamma(X, X)= & \operatorname{Hess} \hat{\gamma}\left(X^{*}, X^{*}\right)-2 \mathcal{H}(h)\langle\nabla \gamma, X\rangle\langle T, X\rangle \\
& +\langle\tilde{\nabla} \tilde{\gamma}, N\rangle\langle A X, X\rangle
\end{aligned}
$$

for every tangent vector field $X \in T \Sigma$.

Observe that, using (16),

$$
|\mathcal{H}(h)\langle\nabla \gamma, X\rangle\langle T, X\rangle| \leq|\mathcal{H}(h)|\|\nabla \gamma\|\|X\|^{2} \leq c \sqrt{\gamma}\|X\|^{2}
$$

for a constant $c>0$, since $|\mathcal{H}(h)| \leq \max _{\left[t_{1}, t_{2}\right]}|\mathcal{H}(t)|$. On the other hand, reasoning as we did before in deriving (12), from condition (13) and using the Hessian comparison theorem for $\mathbb{P}^{n}$ it follows that, if $\gamma$ is sufficiently large, then

$$
\operatorname{Hess} \hat{\gamma}\left(X^{*}, X^{*}\right) \leq c \sqrt{\gamma G(\sqrt{\gamma})}\|X\|^{2}
$$


for a certain positive constant $c$, where we are using the fact that

$$
\left\|X^{*}\right\|_{\mathbb{P}} \leq \frac{1}{\inf _{\Sigma} \rho(h)}\|X\| \leq \frac{1}{\min _{\left[t_{1}, t_{2}\right]} \rho(t)}\|X\| .
$$

Therefore, since $\lim _{t \rightarrow+\infty} G(t)=+\infty$, from (18) we conclude

$$
\operatorname{Hess} \gamma(X, X) \leq c \sqrt{\gamma G(\sqrt{\gamma})}\|X\|^{2}+\langle\tilde{\nabla} \tilde{\gamma}, N\rangle\langle A X, X\rangle
$$

for every tangent vector field $X \in T \Sigma$, outside a compact subset of $\Sigma$.

Assume now that $\sup _{\Sigma}|H|<+\infty$. Tracing (19) we obtain

$$
\Delta \gamma \leq n c \sqrt{\gamma G(\sqrt{\gamma})}+n H\langle\tilde{\nabla} \tilde{\gamma}, N\rangle
$$

outside a compact set. Furthermore, by (16)

$$
|H\langle\tilde{\nabla} \tilde{\gamma}, N\rangle| \leq \sup _{\Sigma}|H|\|\tilde{\nabla} \tilde{\gamma}\| \leq c \sqrt{\gamma} \leq c \sqrt{\gamma G(\sqrt{\gamma})}
$$

for some constant $c>0$. Thus, we conclude that, outside a compact subset of $\Sigma$,

$$
\Delta \gamma \leq c \sqrt{\gamma G(\sqrt{\gamma})}
$$

for some constant $c>0$, which means that condition (4) in Theorem 1 is fulfilled for the Laplacian operator. Therefore, the Omori-Yau maximum principle holds on $\Sigma$ for the Laplacian.

On the other hand, if we assume instead that $\sup _{\Sigma}\|A\|^{2}<+\infty$ then, using again (16), we have

$$
|\langle\tilde{\nabla} \tilde{\gamma}, N\rangle\langle A X, X\rangle| \leq\|\tilde{\nabla} \tilde{\gamma}\|\|A\|\|X\|^{2} \leq c \sqrt{\gamma G(\sqrt{\gamma})}\|X\|^{2}
$$

for a positive constant $c$, if $\gamma$ is sufficiently large. From (19) we therefore obtain

$$
\operatorname{Hess} \gamma(X, X) \leq c \sqrt{\gamma G(\sqrt{\gamma})}\|X\|^{2},
$$

for every tangent vector field $X \in T \Sigma$, outside a compact subset of $\Sigma$. Thus, if $P$ is a positive semi-definite operator with $\sup _{\Sigma} \operatorname{Tr} P<+\infty$, we conclude from here that

$$
L \gamma \leq n c \sup _{\Sigma} \operatorname{Tr} P \sqrt{\gamma G(\sqrt{\gamma})}
$$

if $\gamma$ is sufficiently large, which means that condition (4) in Theorem 1 is fulfilled for the operator $L$. Therefore the Omori-Yau maximum principle holds on $\Sigma$ for $L$. We summarize the above discussion in the following:

Corollary 4. Let $\mathbb{P}^{n}$ be a complete, non-compact, Riemannian manifold whose radial sectional curvature satisfies the condition (13). Let $f: \Sigma^{n} \rightarrow M^{n+1}=I \times{ }_{\rho} \mathbb{P}^{n}$ be a properly immersed hypersurface contained in a slab.

(1) If $\sup _{\Sigma}|H|<+\infty$, then $\Sigma$ is complete and the Omori-Yau maximum principle holds on $\Sigma$ for the Laplacian.

(2) If $\sup _{\Sigma}\|A\|<+\infty$, then $\Sigma$ is complete and the Omori-Yau maximum principle holds on $\Sigma$ for any semi-elliptic operator $L=\operatorname{Tr}(P \circ$ hess $)$ with $\sup _{\Sigma} \operatorname{Tr} P<+\infty$. 
Remark 5. From the equality

$$
\|A\|^{2}=n^{2} H_{1}^{2}-n(n-1) H_{2}
$$

it follows that under the assumption $\inf _{\Sigma} H_{2}>-\infty$ the condition $\sup _{\Sigma}\|A\|^{2}<+\infty$ is equivalent to $\sup _{\Sigma}\left|H_{1}\right|<+\infty$.

\section{Curvature estimates for hypersurfaces in Warped Products}

In this section we will derive some estimates for the $k$-mean curvatures of a hypersurface in a slab of a warped product space. Towards this aim we need the next computational

Proposition 6. Let $f: \Sigma^{n} \rightarrow M^{n+1}=I \times{ }_{\rho} \mathbb{P}^{n}$ be an isometric immersion into a warped product space. Let $h$ be the height function and define

$$
\sigma(t)=\int_{t_{0}}^{t} \rho(u) \mathrm{d} u .
$$

Then

$$
L_{k} h=\mathcal{H}(h)\left(c_{k} H_{k}-\left\langle P_{k} \nabla h, \nabla h\right\rangle\right)+c_{k} \Theta H_{k+1},
$$

and

$$
L_{k} \sigma(h)=c_{k} \rho(h)\left(\mathcal{H}(h) H_{k}+\Theta H_{k+1}\right),
$$

where $c_{k}=(n-k)\left(\begin{array}{l}n \\ k\end{array}\right)=(k+1)\left(\begin{array}{c}n \\ k+1\end{array}\right), \mathcal{H}(t)=\rho^{\prime}(t) / \rho(t)$, and $\Theta=\langle N, T\rangle$ is the angle function.

Proof. The gradient of $\pi_{I} \in C^{\infty}(M)$ is $\bar{\nabla} \pi_{I}=T$, hence,

$$
\nabla h=\left(\bar{\nabla} \pi_{I}\right)^{\top}=T-\Theta N .
$$

Recall that the Levi-Civita connection of a warped product satisfies

$$
\bar{\nabla}_{X} T=\mathcal{H}(X-\langle X, T\rangle T), \quad \text { for any } X \in T M .
$$

Thus

$$
\bar{\nabla}_{X} \nabla h=\mathcal{H}(h)(X-\langle X, T\rangle T)-X(\Theta) N+\Theta A X,
$$

for any $X \in T \Sigma$. Then

$$
\operatorname{hess} h(X)=\nabla_{X} \nabla h=\mathcal{H}(h)(X-\langle X, \nabla h\rangle \nabla h)+\Theta A X,
$$

where $\nabla$ denotes the Levi-Civita connection on $\Sigma^{n}$. Let $\left\{e_{1}, \ldots, e_{n}\right\}$ be a local orthonormal frame on $\Sigma$. Then, using the expressions of the traces of $P_{k}$ and $P_{k} A$

$$
\begin{aligned}
L_{k} h & =\operatorname{Tr}\left(P_{k} \circ \text { hess } h\right)=\sum_{i}\left\langle P_{k} \text { hess } h\left(e_{i}\right), e_{i}\right\rangle \\
& =\mathcal{H}(h)\left(\operatorname{Tr} P_{k}-\left\langle P_{k} \nabla h, \nabla h\right\rangle\right)+\Theta \operatorname{Tr}\left(P_{k} A\right) \\
& =\mathcal{H}(h)\left(c_{k} H_{k}-\left\langle P_{k} \nabla h, \nabla h\right\rangle\right)+c_{k} \Theta H_{k+1} .
\end{aligned}
$$

On the other hand, since $\nabla \sigma(h)=\rho(h) \nabla h$, we have

$$
\text { hess } \sigma(h)(X)=\rho^{\prime}(h)\langle\nabla h, X\rangle \nabla h+\rho(h) \text { hess } h(X) \text {. }
$$


Therefore

$$
\begin{aligned}
L_{k} \sigma(h)= & \operatorname{Tr}\left(P_{k} \circ \operatorname{hess} \sigma(h)\right)=\sum_{i}\left\langle P_{k} \operatorname{hess} \sigma(h)\left(e_{i}\right), e_{i}\right\rangle \\
= & \rho^{\prime}(h)\left\langle P_{k} \nabla h, \nabla h\right\rangle+\rho(h) \mathcal{H}(h)\left(\operatorname{Tr} P_{k}-\left\langle P_{k} \nabla h, \nabla h\right\rangle\right) \\
& +\rho(h) \Theta \operatorname{Tr}\left(P_{k} A\right) \\
= & c_{k} \rho(h)\left(\mathcal{H}(h) H_{k}+\Theta H_{k+1}\right) .
\end{aligned}
$$

As a first application of the computations above, we derive the following:

Theorem 7. Let $f: \Sigma^{n} \rightarrow I \times{ }_{\rho} \mathbb{P}^{n}$ be an immersed hypersurface. If the Omori-Yau maximum principle holds on $\Sigma$ for the Laplacian and $h^{*}=\sup _{\Sigma} h<+\infty$, then

$$
\sup _{\Sigma}|H| \geq \inf _{\Sigma} \mathcal{H}(h) \text {. }
$$

In particular, and as an application of Corollary 4 we deduce the following result, which generalizes Theorem 2 in [3].

Corollary 8. Let $\mathbb{P}^{n}$ be a complete, non-compact, Riemannian manifold whose radial sectional curvature satisfies condition (13). If $f: \Sigma^{n} \rightarrow M^{n+1}=I \times{ }_{\rho} \mathbb{P}^{n}$ is a properly immersed hypersurface contained in a slab, then

$$
\sup _{\Sigma}|H| \geq \inf _{\Sigma} \mathcal{H}(h) .
$$

In other words, there is no properly immersed hypersurface contained in a slab $\left[t_{1}, t_{2}\right] \times \mathbb{P}^{n}$ with

$$
\sup _{\Sigma}|H|<\inf _{\left[t_{1}, t_{2}\right]} \mathcal{H}(t) .
$$

For the proof of Corollary 8 , observe that if $\sup _{\Sigma}|H|=+\infty$, then the inequality (25) trivially holds. On the other hand, if $\sup _{\Sigma}|H|<+\infty$, then by Corollary 4 we know that the Omori-Yau maximum principle holds on $\Sigma$ and the result follows from Theorem 7

Proof of Theorem 7. Since $h$ is bounded from above, we may find a sequence $\left\{q_{j}\right\} \subset$ $\Sigma^{n}$ such that

$$
\begin{aligned}
\lim _{j \rightarrow+\infty} h\left(q_{j}\right) & =h^{*}:=\sup h \\
\left\|\nabla h\left(q_{j}\right)\right\|^{2} & =1-\Theta^{2}\left(q_{j}\right)<\left(\frac{1}{j}\right)^{2}, \\
\Delta h\left(q_{j}\right) & =\mathcal{H}\left(h\left(q_{j}\right)\right)\left(n-\left\|\nabla h\left(q_{j}\right)\right\|^{2}\right)+n H\left(q_{j}\right) \Theta\left(q_{j}\right)<\frac{1}{j} .
\end{aligned}
$$

Then

Making $j \rightarrow+\infty$, we get

$$
\frac{1}{j}>\Delta h\left(q_{j}\right) \geq \mathcal{H}\left(h\left(q_{j}\right)\right)\left(n-\left\|\nabla h\left(q_{j}\right)\right\|^{2}\right)-n \sup _{\Sigma}|H| .
$$

so that

$$
0 \geq \mathcal{H}\left(h^{*}\right)-\sup _{\Sigma}|H|
$$

$$
\sup _{\Sigma}|H| \geq \mathcal{H}\left(h^{*}\right) \geq \inf _{\Sigma} \mathcal{H}(h) .
$$


Corollary 9. Let $\mathbb{P}^{n}$ be a complete, non-compact, Riemannian manifold whose radial sectional curvature satisfies condition (13). If $f: \Sigma^{n} \rightarrow M^{n+1}=I \times \mathrm{e}^{t} \mathbb{P}^{n}$ is a parabolic, properly immersed hypersurface with constant mean curvature $|H| \leq 1$ contained in a slab, then $f(\Sigma)$ is slice.

For the proof of this corollary observe that from Corollary 8 it must be $|H|=1$. Choose the orientation so that $H=1$. In this case $\sigma(h)=\mathrm{e}^{h}$ and by (22)

$$
\Delta \mathrm{e}^{h}=n \mathrm{e}^{h}(1+\Theta) \geq 0 .
$$

Therefore, since $\mathrm{e}^{h} \leq \mathrm{e}^{h^{*}}$ it follows that $\mathrm{e}^{h}$ is a subharmonic function on $\Sigma$ which is bounded from above. The conclusion now follows from parabolicity.

For the next results, we normalize the operators $P_{k}$ to the following

$$
\hat{P}_{k}=\frac{1}{H_{k}} P_{k}
$$

where, of course, we are assuming $H_{k}>0$. We will denote by $\hat{L}_{k}$ the corresponding differential operator, that is

$$
\hat{L}_{k}=\operatorname{Tr}\left(\hat{P}_{k} \circ \text { hess }\right) .
$$

Observe that

$$
\operatorname{Tr}\left(\hat{P}_{k}\right)=c_{k}
$$

so that the operators $\hat{P}_{k}$ have trace always bounded from above. With this preparation we state the next

Theorem 10. Let $f: \Sigma^{n} \rightarrow I \times{ }_{\rho} \mathbb{P}^{n}$ be an immersed hypersurface with $H_{2}>0$. If the Omori-Yau maximum principle holds on $\Sigma$ for $\hat{L}_{1}$ and $h^{*}=\sup _{\Sigma} h<+\infty$, then

$$
\sup _{\Sigma} H_{2}^{1 / 2} \geq \inf _{\Sigma} \mathcal{H}(h)
$$

Proof. We may assume without loss of generality that $\sup _{\Sigma} H_{2}<+\infty$ and $\inf _{\Sigma} \mathcal{H}(h) \geq 0$. Otherwise the desired conclusion trivially holds. Using the basic inequality $H_{1} \geq \sqrt{H_{2}}$ we know that $H_{1}>0$ and $\hat{L}_{1}$ is a well defined elliptic operator. Since $h$ is bounded from above and $\sup _{\Sigma} \sigma(h)=\sigma\left(h^{*}\right)$, we may find a sequence $\left\{q_{j}\right\} \subset \Sigma^{n}$ such that

$$
\begin{aligned}
\lim _{j \rightarrow+\infty}(\sigma \circ h)\left(q_{j}\right) & =\sigma\left(h^{*}\right):=\sup (\sigma \circ h), \\
\left\|\nabla(\sigma \circ h)\left(q_{j}\right)\right\|^{2} & =\rho\left(h\left(q_{j}\right)\right)^{2}\left(1-\Theta^{2}\left(q_{j}\right)\right)<\left(\frac{1}{j}\right)^{2}, \\
\hat{L}_{1}(\sigma \circ h)\left(q_{j}\right) & <\frac{1}{j} .
\end{aligned}
$$

Then we have

$$
\begin{aligned}
\frac{1}{j} & >\hat{L}_{1}(\sigma \circ h)\left(q_{j}\right)=n(n-1) \rho\left(h\left(q_{j}\right)\right)\left(\mathcal{H}\left(h\left(q_{j}\right)\right)+\Theta\left(q_{j}\right) \frac{H_{2}}{H_{1}}\left(q_{j}\right)\right) \\
& \geq n(n-1) \rho\left(h\left(q_{j}\right)\right)\left(\mathcal{H}\left(h\left(q_{j}\right)\right)-\frac{H_{2}}{H_{1}}\left(q_{j}\right)\right) \\
& \geq n(n-1) \rho\left(h\left(q_{j}\right)\right)\left(\mathcal{H}\left(h\left(q_{j}\right)\right)-\sqrt{H_{2}}\left(q_{j}\right)\right) .
\end{aligned}
$$

Observe that

$$
\lim _{j \rightarrow+\infty}(\sigma \circ h)\left(q_{j}\right)=\sigma\left(h^{*}\right):=\sup (\sigma \circ h)
$$


implies $\lim _{j \rightarrow+\infty} h\left(q_{j}\right)=h^{*}$, because $\sigma(t)$ is strictly increasing. Making $j \rightarrow+\infty$, and, if necessary, up to passing to a subsequence we get

$$
0 \geq \mathcal{H}\left(h^{*}\right)-\sup _{\Sigma} \sqrt{H_{2}} \text {. }
$$

So

$$
\sup _{\Sigma} H_{2}^{1 / 2} \geq \mathcal{H}\left(h^{*}\right) \geq \inf _{\Sigma} \mathcal{H}(h)
$$

As a consequence of the previous theorem and of Corollary 4 (see also Remark 5) we have

Corollary 11. Let $\mathbb{P}^{n}$ be a complete, non-compact, Riemannian manifold whose radial sectional curvature satisfies condition (13). If $f: \Sigma^{n} \rightarrow M^{n+1}=I \times{ }_{\rho} \mathbb{P}^{n}$ is a properly immersed hypersurface with $H_{2}>0, \sup _{\Sigma}\left|H_{1}\right|<+\infty$ and contained in a slab, then

$$
\sup _{\Sigma} H_{2}^{1 / 2} \geq \inf _{\Sigma} \mathcal{H}(h)
$$

In other words, there is no properly immersed hypersurface with $\mathrm{H}_{2}>0$ and $\sup _{\Sigma}\left|H_{1}\right|<+\infty$ contained in a slab $\left[t_{1}, t_{2}\right] \times \mathbb{P}^{n}$ with

$$
\sup _{\Sigma} H_{2}^{1 / 2}<\inf _{\left[t_{1}, t_{2}\right]} \mathcal{H}(t) \text {. }
$$

In the next theorem, the existence of an elliptic point enable us to guarantee that $H_{k-1}$ is strictly positive and to guarantee ellipticity of the operator $\hat{L}_{k-1}$. Reasoning as in the previous results gives the next

Theorem 12. Let $f: \Sigma^{n} \rightarrow I \times{ }_{\rho} \mathbb{P}^{n}$ be an immersed hypersurface having an elliptic point, with $H_{k}>0$. If the Omori-Yau maximum principle holds on $\Sigma$ for $\hat{L}_{k-1}$, with $3 \leq k \leq n$, and $h^{*}<+\infty$ then

$$
\sup _{\Sigma} H_{k}^{1 / k} \geq \inf _{\Sigma} \mathcal{H}(h) \text {. }
$$

Corollary 13. Let $\mathbb{P}^{n}$ be a complete, non-compact, Riemannian manifold whose radial sectional curvature satisfies condition (13). Assume that $f: \Sigma^{n} \rightarrow M^{n+1}=$ $I \times{ }_{\rho} \mathbb{P}^{n}$ is a properly immersed hypersurface having an elliptic point, with $H_{k}>0$ and $\sup _{\Sigma}\left|H_{1}\right|<+\infty$. If $f(\Sigma)$ is contained in a slab, then

$$
\sup _{\Sigma} H_{k}^{1 / k} \geq \inf _{\Sigma} \mathcal{H}(h)
$$

for every $3 \leq k \leq n$. In other words, there is no properly immersed hypersurface having an elliptic point, with $H_{k}>0, \sup _{\Sigma}\left|H_{1}\right|<+\infty$ and contained in a slab $\left[t_{1}, t_{2}\right] \times \mathbb{P}^{n}$ with

$$
\sup _{\Sigma} H_{k}^{1 / k}<\inf _{\left[t_{1}, t_{2}\right]} \mathcal{H}(t)
$$

\section{Hypersurfaces With CONSTANT 2-MEAn CURVATURE}

In this section we will derive some applications for hypersurfaces with positive constant 2-mean curvature $\mathrm{H}_{2}$. Before stating the main results, let us introduce an auxiliary lemma that will be useful in the sequel. 
Lemma 14. Let $f: \Sigma^{n} \rightarrow I \times{ }_{\rho} \mathbb{P}^{n}$ be a hypersurface with non-vanishing mean curvature which is contained in a slab. Assume that $\mathcal{H}^{\prime} \geq 0$ and that the angle function $\Theta$ does not change sign. Choose on $\Sigma$ the orientation so that $H_{1}>0$. Suppose the Omori-Yau maximum principle for the Laplacian holds on $\Sigma$. We have that

$$
\begin{aligned}
\text { (i) } \text { if } \Theta \leq 0, & \text { then } \mathcal{H}(h) \geq 0, \\
\text { (ii) } \text { if } \Theta \geq 0, & \text { then } \mathcal{H}(h) \leq 0 .
\end{aligned}
$$

Proof. Since $h$ is bounded from below and the Omori-Yau maximum principle for the Laplacian operator holds on $\Sigma$, we can find a sequence $\left\{p_{j}\right\} \subset \Sigma^{n}$ such that

$$
\begin{aligned}
\lim _{j \rightarrow+\infty} h\left(p_{j}\right) & =h_{*}:=\inf h, \\
\left\|\nabla h\left(p_{j}\right)\right\|^{2} & =1-\Theta^{2}\left(p_{j}\right)<\left(\frac{1}{j}\right)^{2}, \\
\Delta h\left(p_{j}\right) & =\mathcal{H}\left(h\left(p_{j}\right)\right)\left(n-\left\|\nabla h\left(p_{j}\right)\right\|^{2}\right)+n H_{1}\left(p_{j}\right) \Theta\left(p_{j}\right)>-\frac{1}{j} .
\end{aligned}
$$

Then

$$
-n H_{1}\left(p_{j}\right) \Theta\left(p_{j}\right)<\frac{1}{j}+\mathcal{H}\left(h\left(p_{j}\right)\right)\left(n-\left\|\nabla h\left(p_{j}\right)\right\|^{2}\right) .
$$

Similarly, since $h$ is bounded from above, we can also find a second sequence $\left\{q_{j}\right\} \subset$ $\Sigma^{n}$ such that

$$
\begin{aligned}
\lim _{j \rightarrow+\infty} h\left(q_{j}\right) & =h^{*}:=\sup h \\
\left\|\nabla h\left(q_{j}\right)\right\|^{2} & =1-\Theta^{2}\left(q_{j}\right)<\left(\frac{1}{j}\right)^{2}, \\
\Delta h\left(q_{j}\right) & =\mathcal{H}\left(h\left(q_{j}\right)\right)\left(n-\left\|\nabla h\left(q_{j}\right)\right\|^{2}\right)+n H_{1}\left(q_{j}\right) \Theta\left(q_{j}\right)<\frac{1}{j} .
\end{aligned}
$$

Then

$$
-n H_{1}\left(q_{j}\right) \Theta\left(q_{j}\right)>-\frac{1}{j}+\mathcal{H}\left(h\left(q_{j}\right)\right)\left(n-\left\|\nabla h\left(q_{j}\right)\right\|^{2}\right) .
$$

Assume first that $\Theta \leq 0$. Since $\lim _{j \rightarrow+\infty}-\Theta\left(p_{j}\right)=-\operatorname{sgn} \Theta=1>0$, we have $-\Theta\left(p_{j}\right)>0$ for sufficiently large $j$. Since $H_{1}\left(p_{j}\right)>0$, using (26) it follows from (26) that

$$
0 \leq \liminf _{j \rightarrow+\infty}\left(-H_{1}\left(p_{j}\right) \Theta\left(p_{j}\right)\right) \leq \mathcal{H}\left(h_{*}\right) .
$$

Therefore $\mathcal{H}\left(h_{*}\right) \geq 0$ and, by $\mathcal{H}^{\prime} \geq 0$, we conclude that

$$
\mathcal{H}(h) \geq \mathcal{H}\left(h_{*}\right) \geq 0 .
$$

Assume now that $\Theta \geq 0$; then $\lim _{j \rightarrow+\infty} \Theta\left(q_{j}\right)=\operatorname{sgn} \Theta=1>0$, so that $\Theta\left(q_{j}\right)>0$ for sufficiently large $j$. Therefore, since $H_{1}\left(q_{j}\right)>0$, from (27) we deduce

$$
0 \leq \liminf _{j \rightarrow+\infty}\left(H_{1}\left(q_{j}\right) \Theta\left(q_{j}\right)\right) \leq-\mathcal{H}\left(h^{*}\right) .
$$

Therefore $\mathcal{H}\left(h^{*}\right) \leq 0$ and, by $\mathcal{H}^{\prime} \geq 0$, we conclude that

$$
\mathcal{H}(h) \leq \mathcal{H}\left(h^{*}\right) \leq 0 .
$$

This concludes the proof. 
In the rest of this section we will work basically with the operator $L_{1}$. We will assume that $H_{2}$ is a positive constant. Recall that this implies, in this case, that the immersion is two sided. We can choose the normal unit vector $N$ on $\Sigma$ such that $H_{1}>0$ and the operator $L_{1}$ is elliptic (see the discussion in the Preliminaries).

Let $\sigma(t)=\int_{t_{0}}^{t} \rho(s) \mathrm{d} s$. By Proposition 6 we know that

$$
\begin{aligned}
\Delta \sigma(h) & =n \rho(h)\left(\mathcal{H}(h)+\Theta H_{1}\right), \\
L_{1} \sigma(h) & =n(n-1) \rho(h)\left(\mathcal{H}(h) H_{1}+\Theta H_{2}\right) .
\end{aligned}
$$

Therefore,

$$
\mathcal{L}_{1} \sigma(h)=n(n-1) \rho(h)\left(\mathcal{H}(h)^{2}-\Theta^{2} H_{2}\right),
$$

where $\mathcal{L}_{1}$ is the operator given by

$$
\mathcal{L}_{1}=(n-1) \mathcal{H}(h) \Delta-\Theta L_{1}=\operatorname{Tr}\left(\mathcal{P}_{1} \circ \text { hess }\right),
$$

with

$$
\mathcal{P}_{1}=(n-1) \mathcal{H}(h) I-\Theta P_{1} .
$$

Let us now state the first main result of this section, which extends Theorem 2.4 in [4] to the case of constant 2-mean curvature $\mathrm{H}_{2}$.

Theorem 15. Let $f: \Sigma^{n} \rightarrow I \times{ }_{\rho} \mathbb{P}^{n}$ be a compact hypersurface of constant positive 2-mean curvature $H_{2}$. If $\mathcal{H}^{\prime}(t) \geq 0$ and the angle function $\Theta$ does not change sign, then $\mathbb{P}^{n}$ is necessarily compact and $f\left(\Sigma^{n}\right)$ is a slice.

Proof. As indicated above, we choose the orientation of $\Sigma$ so that $H_{1}>0$. Since $\Sigma^{n}$ is compact, we may apply Lemma 14. Let us consider first the case where $\Theta \leq 0$, for which $\mathcal{H}(h) \geq 0$. Thus, the operator $\mathcal{P}_{1}$ is positive semi-definite or, equivalently, $\mathcal{L}_{1}$ is semi-elliptic.

Since $\Sigma$ is compact, there exist points $p_{\max } \in \Sigma$ and $p_{\min } \in \Sigma$ such that

$$
h\left(p_{\max }\right)=h^{*}=\max _{\Sigma} h \text { and } h\left(p_{\min }\right)=h_{*}=\min _{\Sigma} h .
$$

Therefore, $\left\|\nabla h\left(p_{\max }\right)\right\|=\left\|\nabla h\left(p_{\min }\right)\right\|=0$, which yields

$$
\Theta\left(p_{\max }\right)=\Theta\left(p_{\min }\right)=-1
$$

because of (23). Observe that

$$
(\sigma \circ h)^{*}=\max _{\Sigma}(\sigma \circ h)=\sigma\left(h^{*}\right)=\sigma\left(h\left(p_{\max }\right)\right)
$$

and

$$
(\sigma \circ h)_{*}=\min _{\Sigma}(\sigma \circ h)=\sigma\left(h_{*}\right)=\sigma\left(h\left(p_{\min }\right)\right),
$$

because $\sigma(t)$ is strictly increasing. In particular,

$$
\operatorname{Hess} \sigma(h)\left(p_{\max }\right) \leq 0 \text { and } \operatorname{Hess} \sigma(h)\left(p_{\min }\right) \geq 0 .
$$

Taking into account that $\mathcal{P}_{1}$ is positive semi-definite, yields

$$
\mathcal{L}_{1} \sigma(h)\left(p_{\text {max }}\right)=n(n-1) \rho\left(h^{*}\right)\left(\mathcal{H}\left(h^{*}\right)^{2}-H_{2}\right) \leq 0
$$

and

$$
\mathcal{L}_{1} \sigma(h)\left(p_{\text {min }}\right)=n(n-1) \rho\left(h_{*}\right)\left(\mathcal{H}\left(h_{*}\right)^{2}-H_{2}\right) \geq 0 .
$$

Then, since $\mathcal{H}(h) \geq 0$ on $\Sigma$, we obtain

$$
\mathcal{H}\left(h_{*}\right) \geq H_{2}^{1 / 2} \geq \mathcal{H}\left(h^{*}\right) .
$$


On the other hand, by $\mathcal{H}^{\prime} \geq 0$ we also have $\mathcal{H}\left(h_{*}\right) \leq \mathcal{H}\left(h^{*}\right)$. Thus the validity of the equality $\mathcal{H}\left(h_{*}\right)=\mathcal{H}\left(h^{*}\right)$ and $\mathcal{H}(h)=H_{2}^{1 / 2}$ is constant on $\Sigma$. By (28), using the basic inequality $H_{1} \geq H_{2}^{1 / 2}$ and the fact that $\Theta \geq-1$, we obtain

$$
\begin{aligned}
L_{1} \sigma(h) & =n(n-1) \rho(h) H_{2}^{1 / 2}\left(H_{1}+\Theta H_{2}^{1 / 2}\right) \\
& \geq n(n-1) \rho(h) H_{2}^{1 / 2}\left(H_{1}-H_{2}^{1 / 2}\right) \geq 0 .
\end{aligned}
$$

That is, $L_{1} \sigma(h) \geq 0$ on the compact manifold $\Sigma$. Thus, by the maximum principle applied to the elliptic operator $L_{1}$, we conclude that $\sigma(h)$, and hence $h$, is constant.

Finally, in the case where $\Theta \geq 0$, we know from Lemma 14 that $\mathcal{H}(h) \leq 0$ on $\Sigma$, so that the operator $-\mathcal{L}_{1}$ is semi-elliptic. The proof then follows as in the case $\Theta \leq 0$, working with $-\mathcal{L}_{1}$ instead of $\mathcal{L}_{1}$.

In our next result, we consider the case of complete (and non-compact) hypersurfaces, extending Theorem 2.9 in 4 to the case of constant 2-mean curvature hypersurfaces.

Theorem 16. Let $f: \Sigma^{n} \rightarrow I \times{ }_{\rho} \mathbb{P}^{n}$ be a complete hypersurface of constant positive 2-mean curvature $\mathrm{H}_{2}$ such that

$$
K_{\Sigma}^{\mathrm{rad}} \geq-G(r)
$$

Here $G$ is a smooth function on $[0,+\infty)$ which is even at the origin and satisfies conditions (i)-(iv) listed in Theorem 1. Assume that $\sup _{\Sigma}\left|H_{1}\right|<+\infty$ and that $\Sigma$ is contained in a slab, that is,

$$
f\left(\Sigma^{n}\right) \subset\left[t_{1}, t_{2}\right] \times \mathbb{P}^{n},
$$

where $t_{1}, t_{2} \in I$ are finite. If $\mathcal{H}^{\prime}(t)>0$ almost everywhere and the angle function $\Theta$ does not change sign, then $f\left(\Sigma^{n}\right)$ is a slice.

Proof. Choose the orientation of $\Sigma$ so that $H_{1}>0$. By Corollary 3 we know that the Omori-Yau maximum principle holds on $\Sigma$ for the Laplacian operator, so that we may apply Lemma 14 .

In the case where $\Theta \leq 0$, by Lemma 14 we have $\mathcal{H}(h) \geq 0$, and therefore the operator $\mathcal{P}_{1}$ is positive semi-definite. In other words, the differential operator $\mathcal{L}_{1}$ is semi-elliptic. Furthermore

$$
\operatorname{Tr} \mathcal{P}_{1}=n(n-1) \mathcal{H}(h)-n(n-1) H_{1} \Theta \leq n(n-1)\left(\mathcal{H}\left(h^{*}\right)+H_{1}^{*}\right),
$$

where $h^{*}=\sup _{\Sigma} h<+\infty$ and $H_{1}^{*}=\sup _{\Sigma} H_{1}<+\infty$. Hence by Corollary 3 we know that the Omori-Yau maximum principle holds on $\Sigma$ for the operator $\mathcal{L}_{1}$.

Since $\sup _{\Sigma} \sigma(h)=\sigma\left(h^{*}\right)<+\infty$, there exists a sequence $\left\{p_{j}\right\}_{j \in \mathbb{N}} \subset \Sigma$ such that

$$
\begin{aligned}
& \text { (i) } \lim _{j \rightarrow+\infty} \sigma\left(h\left(p_{j}\right)\right)=\sup _{\Sigma} \sigma(h)=\sigma\left(h^{*}\right), \\
& \text { (ii) }\left\|\nabla(\sigma \circ h)\left(p_{j}\right)\right\|=\rho\left(h\left(p_{j}\right)\right)\left\|\nabla h\left(p_{j}\right)\right\|<\frac{1}{j}, \\
& \text { (iii) } \mathcal{L}_{1}(\sigma \circ h)\left(p_{j}\right)<\frac{1}{j} .
\end{aligned}
$$

Observe that condition (i) implies that $\lim _{j \rightarrow+\infty} h\left(p_{j}\right)=h^{*}$, because $\sigma(t)$ is strictly increasing. Thus by condition (ii) we also have $\lim _{j \rightarrow+\infty}\left\|\nabla h\left(p_{j}\right)\right\|=0$. Therefore

$$
\mathcal{L}_{1} \sigma(h)\left(p_{j}\right)=n(n-1) \rho\left(h\left(p_{j}\right)\right)\left(\mathcal{H}\left(h\left(p_{j}\right)\right)^{2}-\Theta^{2}\left(p_{j}\right) H_{2}\right)<\frac{1}{j},
$$


and taking the limit for $j \rightarrow+\infty$ and observing that $\Theta^{2}\left(p_{j}\right)=1-\left\|\nabla h\left(p_{j}\right)\right\|^{2} \rightarrow 1$ as $j \rightarrow+\infty$, we find

$$
\mathcal{H}\left(h^{*}\right)^{2}-H_{2} \leq 0 \text {. }
$$

On the other hand, since $h$ is also bounded from below, $\inf _{\Sigma} \sigma(h)=\sigma\left(h_{*}\right)>-\infty$, where $h_{*}=\inf _{\Sigma} h>-\infty$. Thus, we can find a sequence $\left\{q_{j}\right\}_{j \in \mathbb{N}} \subset \Sigma$ such that

$$
\begin{aligned}
& \text { (i) } \lim _{j \rightarrow+\infty} \sigma\left(h\left(q_{j}\right)\right)=\inf _{\Sigma} \sigma(h)=\sigma\left(h_{*}\right), \\
& \text { (ii) }\left\|\nabla(\sigma \circ h)\left(q_{j}\right)\right\|=\rho\left(h\left(q_{j}\right)\right)\left\|\nabla h\left(q_{j}\right)\right\|<\frac{1}{j}, \\
& \text { (iii) } \mathcal{L}_{1}(\sigma \circ h)\left(q_{j}\right)>-\frac{1}{j} .
\end{aligned}
$$

Hence, proceeding as above and using that

$$
\mathcal{L}_{1} \sigma(h)\left(q_{j}\right)=n(n-1) \rho\left(h\left(q_{j}\right)\right)\left(\mathcal{H}\left(h\left(q_{j}\right)\right)^{2}-\Theta^{2}\left(q_{j}\right) H_{2}\right)>-\frac{1}{j},
$$

we find

$$
\mathcal{H}\left(h_{*}\right)^{2}-H_{2} \geq 0
$$

Thus $\mathcal{H}\left(h_{*}\right)^{2} \geq \mathcal{H}\left(h^{*}\right)^{2}$ and, taking into account that $\mathcal{H}\left(h_{*}\right), \mathcal{H}\left(h^{*}\right) \geq 0$, this gives $\mathcal{H}\left(h_{*}\right) \geq \mathcal{H}\left(h^{*}\right)$. Therefore, since $\mathcal{H}(t)$ is an increasing function, we conclude that $h^{*}=h_{*}$.

Finally, let us consider the case where $\Theta \geq 0$. By Lemma 14 we find that $\mathcal{H}(h) \leq 0$ and then the operator $-\mathcal{L}_{1}$ is semi-elliptic. Moreover

$$
\operatorname{Tr}\left(-\mathcal{P}_{1}\right)=-n(n-1) \mathcal{H}(h)+n(n-1) H_{1} \Theta \leq n(n-1)\left(-\mathcal{H}\left(h_{*}\right)+H_{1}^{*}\right) .
$$

Hence the trace of $-\mathcal{P}_{1}$ is bounded from above and by Corollary 3 the Omori-Yau maximum principle holds for the operator $-\mathcal{L}_{1}$. Proceeding as above, we arrive at the two inequalities

$$
H_{2}-\mathcal{H}\left(h_{*}\right)^{2} \geq 0 \quad \text { and } \quad H_{2}-\mathcal{H}\left(h^{*}\right)^{2} \leq 0 .
$$

Thus $\mathcal{H}\left(h_{*}\right)^{2} \leq \mathcal{H}\left(h^{*}\right)^{2}$. Since $\mathcal{H}\left(h_{*}\right), \mathcal{H}\left(h^{*}\right) \leq 0$, this implies $\mathcal{H}\left(h_{*}\right) \geq \mathcal{H}\left(h^{*}\right)$. But $\mathcal{H}(t)$ being increasing, this gives $h_{*}=h^{*}$ concluding the proof.

In particular, Theorem 16 remains true if we replace condition (30) by the stronger condition of $\Sigma^{n}$ having radial sectional curvature bounded from below by a constant. This happens, for instance, when the sectional curvature of $\mathbb{P}^{n}$ is itself bounded from below. This observation yields the next

Corollary 17. Let $\mathbb{P}^{n}$ be a complete Riemannian manifold with sectional curvature bounded from below, and let $f: \Sigma^{n} \rightarrow I \times_{\rho} \mathbb{P}^{n}$ be a complete hypersurface of constant positive 2-mean curvature $H_{2}$. Assume that $\sup _{\Sigma}\left|H_{1}\right|<+\infty$ and that $\Sigma$ is contained in a slab, that is,

$$
f\left(\Sigma^{n}\right) \subset\left[t_{1}, t_{2}\right] \times \mathbb{P}^{n},
$$

where $t_{1}, t_{2} \in I$ are finite. If $\mathcal{H}^{\prime}(t)>0$ almost everywhere and the angle function $\Theta$ does not change sign, then $f\left(\Sigma^{n}\right)$ is a slice.

As already observed, for the proof of Corollary [17 it suffices to show that $K_{\Sigma}^{\mathrm{rad}}$ is bounded from below by a constant, and the result then follows from Theorem 16. Actually, we can prove the following stronger result, which will be useful in the sequel. 
Lemma 18. Let $\mathbb{P}^{n}$ be a Riemannian manifold with sectional curvature bounded from below, and let $f: \Sigma^{n} \rightarrow I \times{ }_{\rho} \mathbb{P}^{n}$ be an immersed hypersurface. Assume that $\sup _{\Sigma}\|A\|^{2}<+\infty$ and that $\Sigma$ is contained in a slab. Then the sectional curvature of $\Sigma$ is bounded from below by a constant.

Given the validity of Lemma 18 and taking into account the equality

$$
\|A\|^{2}=\operatorname{Tr}\left(A^{2}\right)=n^{2} H_{1}^{2}-n(n-1) H_{2},
$$

it follows that, under the assumptions of Corollary 17

$$
\sup _{\Sigma}\|A\|^{2} \leq n^{2}\left(\sup _{\Sigma} H_{1}\right)^{2}-n(n-1) H_{2}<+\infty \text {. }
$$

Thus $K_{\Sigma}^{\mathrm{rad}}$ is bounded from below by a constant.

Proof of Lemma [18, Recall that the Gauss equation for a hypersurface $f: \Sigma^{n} \rightarrow$ $M^{n+1}$ is given by

$$
\langle\mathrm{R}(X, Y) Z, V\rangle=\langle\overline{\mathrm{R}}(X, Y) Z, V\rangle-\langle A Y, V\rangle\langle A X, Z\rangle+\langle A X, V\rangle\langle A Y, Z\rangle,
$$

for $X, Y, Z, V \in T \Sigma$, where $\mathrm{R}$ and $\overline{\mathrm{R}}$ are the curvature tensors of $\Sigma^{n}$ and $M^{n+1}$, respectively. Then, if $\{X, Y\}$ is an orthonormal basis for an arbitrary 2-plane tangent to $\Sigma$, we have

$$
\begin{aligned}
K_{\Sigma}(X, Y) & =\bar{K}(X, Y)+\langle A X, X\rangle\langle A Y, Y\rangle-\langle A X, Y\rangle^{2} \\
& \geq \bar{K}(X, Y)-\|A X\|\|A Y\|-\|A X\|^{2} \\
& \geq \bar{K}(X, Y)-2\|A\|^{2}
\end{aligned}
$$

where the last inequality follows from the fact that

$$
\|A X\|^{2} \leq \operatorname{Tr}\left(A^{2}\right)\|X\|^{2}=\|A\|^{2}
$$

for every unit vector $X$ tangent to $\Sigma$. Since we are assuming that $\sup _{\Sigma}\|A\|^{2}<+\infty$, it suffices to have $\bar{K}(X, Y)$ bounded from below.

The curvature tensor of $M^{n+1}$ expressed in terms of the curvature tensor of $\mathbb{P}^{n}$ is

$$
\begin{aligned}
\overline{\mathrm{R}}(U, V) W= & \mathrm{R}_{\mathbb{P}}\left(U^{*}, V^{*}\right) W^{*}-\mathcal{H}^{2}\left(\pi_{I}\right)(\langle V, W\rangle U-\langle U, W\rangle V) \\
& +\mathcal{H}^{\prime}\left(\pi_{I}\right)\langle W, T\rangle(\langle U, T\rangle V-\langle V, T\rangle U) \\
& -\mathcal{H}^{\prime}\left(\pi_{I}\right)(\langle V, W\rangle\langle U, T\rangle-\langle U, W\rangle\langle V, T\rangle) T,
\end{aligned}
$$

for every $U, V, W \in T M$, where $T=\partial_{t}$ and we are using the notation $U^{*}$ to denote $\pi_{\mathbb{P}_{*}} U$ for an arbitrary $U \in T M$. Then, for the orthonormal basis $\{X, Y\}$ we find that

$$
\begin{aligned}
\bar{K}(X, Y)= & \frac{1}{\rho^{2}(h)} K_{\mathbb{P}}\left(X^{*}, Y^{*}\right)\left\|X^{*} \wedge Y^{*}\right\|^{2} \\
& -\mathcal{H}^{2}(h)-\mathcal{H}^{\prime}(h)\left(\langle X, \nabla h\rangle^{2}+\langle Y, \nabla h\rangle^{2}\right) \\
\geq & \frac{1}{\rho^{2}(h)} K_{\mathbb{P}}\left(X^{*}, Y^{*}\right)\left\|X^{*} \wedge Y^{*}\right\|^{2}-\mathcal{H}^{2}(h)-\left|\mathcal{H}^{\prime}(h)\right|,
\end{aligned}
$$

since

$$
\langle X, \nabla h\rangle^{2}+\langle Y, \nabla h\rangle^{2} \leq\|\nabla h\|^{2} \leq 1
$$


On the other hand,

$$
\begin{aligned}
\left\|X^{*} \wedge Y^{*}\right\|^{2} & =\left\|X^{*}\right\|^{2}\left\|Y^{*}\right\|^{2}-\left\langle X^{*}, Y^{*}\right\rangle^{2} \\
& =1-\langle X, T\rangle^{2}-\langle Y, T\rangle^{2} \leq 1 .
\end{aligned}
$$

Therefore, if $K_{\mathbb{P}} \geq c$ for some constant $c$, we deduce

$$
\frac{1}{\rho^{2}(h)} K_{\mathbb{P}}\left(X^{*}, Y^{*}\right)\left\|X^{*} \wedge Y^{*}\right\|^{2} \geq-\frac{|c|}{\rho^{2}(h)} .
$$

Finally, since $h$ is a bounded function, we conclude from (31), (32), and (33) that the sectional curvature $K(X, Y)$ is bounded from below by an absolute constant.

We observe that condition (30) has been used in the proof of Theorem 16 only to guarantee that the Omori-Yau maximum principle holds on $\Sigma$ for the Laplacian and for the semi-elliptic operator $\mathcal{L}_{1}\left(\right.$ or $\left.-\mathcal{L}_{1}\right)$. Therefore, the theorem remains true under any other hypothesis guaranteeing this latter fact. Thus, and as a consequence of Corollary 4 , we can also state the following:

Theorem 19. Let $\mathbb{P}^{n}$ be a complete, non-compact, Riemannian manifold whose radial sectional curvature satisfies condition (13). Let $f: \Sigma^{n} \rightarrow I \times{ }_{\rho} \mathbb{P}^{n}$ be a properly immersed hypersurface of constant positive 2-mean curvature $\mathrm{H}_{2}$. Assume that $\sup _{\Sigma}\left|H_{1}\right|<+\infty$ and that $\Sigma$ is contained in a slab. If $\mathcal{H}^{\prime}(t)>0$ almost everywhere and the angle function $\Theta$ does not change sign, then $f\left(\Sigma^{n}\right)$ is a slice.

As pointed out before, for the validity of Theorem 19 it suffices to show that the Omori-Yau maximum principle holds on $\Sigma$ for the Laplacian and for the semielliptic operator $\mathcal{L}_{1}\left(\right.$ or $\left.-\mathcal{L}_{1}\right)$. But this follows directly from Corollary 4 , since $\|A\|^{2}=\operatorname{Tr}\left(A^{2}\right)=n^{2} H_{1}^{2}-n(n-1) H_{2}$ and

$$
\sup _{\Sigma}\|A\|^{2} \leq n^{2}\left(\sup _{\Sigma} H_{1}\right)^{2}-n(n-1) H_{2}<+\infty \text {. }
$$

See Remark 5 following Corollary 4

\section{Hypersurfaces With CONSTANT higher order MEAN CURVATURE}

In this section we will extend our previous results to the case of hypersurfaces with non-zero constant $k$-mean curvature $H_{k}$, when $k \geq 3$. To this end, we will work with the operator $L_{k-1}$, and we will assume that there exists an elliptic point in $\Sigma$. Note that the existence of an elliptic point is always guaranteed when $\Sigma$ is compact and $\rho^{\prime} \neq 0$ on $\Sigma$ (see the proof of Theorem 24 below and Lemma 5.3 in [2] in a Lorentzian ambient space). Recall from the discussion in the Preliminaries that the existence of an elliptic point implies that $H_{k}$ is positive, the immersion is two sided and $H_{1}>0$ for the chosen orientation. Moreover, it implies also that for every $1 \leq j \leq k-1$, the operators $L_{j}$ are elliptic or, equivalently, the operators $P_{j}$ are positive definite.

In order to extend our previous results to the case of higher order mean curvatures, we introduce a family of operators, extending $\mathcal{L}_{1}$. For $2 \leq k \leq n$, we define the operator

$$
\mathcal{L}_{k-1}=\operatorname{Tr}\left(\left[\sum_{j=0}^{k-1}(-1)^{j} \frac{c_{k-1}}{c_{j}} \mathcal{H}(h)^{k-1-j} \Theta^{j} P_{j}\right] \circ \text { hess }\right)=\operatorname{Tr}\left(\mathcal{P}_{k-1} \circ \text { hess }\right),
$$


where

$$
\mathcal{P}_{k-1}=\sum_{j=0}^{k-1}(-1)^{j} \frac{c_{k-1}}{c_{j}} \mathcal{H}(h)^{k-1-j} \Theta^{j} P_{j} .
$$

We claim that

$$
\mathcal{L}_{k-1} \sigma(h)=c_{k-1} \rho(h)\left(\mathcal{H}(h)^{k}+(-1)^{k-1} \Theta^{k} H_{k}\right),
$$

and we prove the claim by induction. We have already seen in (29) that the claim is true for $k=2$. For $k \geq 3$, we observe that

$$
\mathcal{P}_{k-1}=\frac{c_{k-1}}{c_{k-2}} \mathcal{H}(h) \mathcal{P}_{k-2}+(-1)^{k-1} \Theta^{k-1} P_{k-1}
$$

and then

$$
\mathcal{L}_{k-1}=\frac{c_{k-1}}{c_{k-2}} \mathcal{H}(h) \mathcal{L}_{k-2}+(-1)^{k-1} \Theta^{k-1} L_{k-1},
$$

Therefore, if $k \geq 3$ and we assume that the claim is true for $\mathcal{L}_{k-2}$, then using (22) we conclude that

$$
\begin{aligned}
\mathcal{L}_{k-1} \sigma(h)= & \frac{c_{k-1}}{c_{k-2}} \mathcal{H}(h) \mathcal{L}_{k-2} \sigma(h)+(-1)^{k-1} \Theta^{k-1} L_{k-1} \sigma(h) \\
= & c_{k-1} \rho(h)\left(\mathcal{H}(h)^{k}+(-1)^{k-2} \mathcal{H}(h) \Theta^{k-1} H_{k-1}\right. \\
& \left.+(-1)^{k-1} \mathcal{H}(h) \Theta^{k-1} H_{k-1}+(-1)^{k-1} \Theta^{k} H_{k}\right) \\
= & c_{k-1} \rho(h)\left(\mathcal{H}(h)^{k}+(-1)^{k-1} \Theta^{k} H_{k}\right) .
\end{aligned}
$$

We are now ready to give the following extension of Theorem 15 .

Theorem 20. Let $f: \Sigma^{n} \rightarrow I \times_{\rho} \mathbb{P}^{n}$ be a compact hypersurface with constant $k$ mean curvature $H_{k}$, with $3 \leq k \leq n$. Assume that there exists an elliptic point in $\Sigma$. If $\mathcal{H}^{\prime}(t) \geq 0$ and the angle function $\Theta$ does not change sign, then $\mathbb{P}^{n}$ is necessarily compact and $f\left(\Sigma^{n}\right)$ is a slice.

Proof. Choose the orientation of $\Sigma$ so that $H_{1}>0$. Since $\Sigma^{n}$ is compact, we may apply Lemma 14. Let us consider first the case when $\Theta \leq 0$, so that $\mathcal{H}(h) \geq 0$. Thus, by (34) the operator $\mathcal{P}_{k-1}$ is positive semi-definite or, equivalently, $\mathcal{L}_{k-1}$ is semi-elliptic. Reasoning as in the proof of Theorem 15, yields

$$
\mathcal{L}_{k-1} \sigma(h)\left(p_{\max }\right)=c_{k-1} \rho\left(h^{*}\right)\left(\mathcal{H}\left(h^{*}\right)^{k}-H_{k}\right) \leq 0
$$

and

$$
\mathcal{L}_{k-1} \sigma(h)\left(p_{\min }\right)=c_{k-1} \rho\left(h_{*}\right)\left(\mathcal{H}\left(h_{*}\right)^{k}-H_{k}\right) \geq 0
$$

with $p_{\max } \in \Sigma$ and $p_{\min } \in \Sigma$ such that $h\left(p_{\max }\right)=h^{*}=\max _{\Sigma} h$ and $h\left(p_{\min }\right)=h_{*}=$ $\min _{\Sigma} h$.

Then, since $\mathcal{H}(h) \geq 0$ on $\Sigma$, we obtain

$$
\mathcal{H}\left(h_{*}\right) \geq H_{k}^{1 / k} \geq \mathcal{H}\left(h^{*}\right) .
$$

On the other hand, by $\mathcal{H}^{\prime} \geq 0$ we also have $\mathcal{H}\left(h_{*}\right) \leq \mathcal{H}\left(h^{*}\right)$. Thus, we have the equality $\mathcal{H}\left(h_{*}\right)=\mathcal{H}\left(h^{*}\right)$ and $\mathcal{H}(h)=H_{k}^{1 / k}$ is constant on $\Sigma$. Therefore, by (22) and using the Gårding inequality $H_{k-1} \geq H_{k}^{(k-1) / k}$ (see (1)) and the fact that $\Theta \geq-1$, we obtain

$$
\begin{aligned}
L_{k-1} \sigma(h) & =c_{k-1} \rho(h) H_{k}^{1 / k}\left(H_{k-1}+\Theta H_{k}^{(k-1) / k}\right) \\
& \geq c_{k-1} \rho(h) H_{k}^{1 / k}\left(H_{k-1}-H_{k}^{(k-1) / k}\right) \geq 0 .
\end{aligned}
$$


That is, $L_{k-1} \sigma(h) \geq 0$ on the compact manifold $\Sigma$. Therefore, by the maximum principle applied to the elliptic operator $L_{k-1}$, we conclude that $\sigma(h)$, and hence $h$, is constant.

Finally, in the case where $\Theta \geq 0$, we know from Lemma 14 that $\mathcal{H}(h) \leq 0$ on $\Sigma$, so that the operator $(-1)^{k-1} \mathcal{L}_{k-1}$ is semi-elliptic. The proof then follows as in the case $\Theta \leq 0$, working with $(-1)^{k-1} \mathcal{L}_{k-1}$ instead.

For the case of complete (and non-compact) hypersurfaces, we can state the following extension of Theorem 16.

Theorem 21. Let $f: \Sigma^{n} \rightarrow I \times{ }_{\rho} \mathbb{P}^{n}$ be a complete hypersurface with constant $k$-mean curvature $H_{k}, 3 \leq k \leq n$, which satisfies condition (30). Assume that there exists an elliptic point in $\Sigma, \sup _{\Sigma}\left|H_{1}\right|<+\infty$ and $\Sigma$ is contained in a slab. If $\mathcal{H}^{\prime}(t)>0$ almost everywhere and the angle function $\Theta$ does not change sign, then $f\left(\Sigma^{n}\right)$ is a slice.

Proof. By Corollary 3, we know that the Omori-Yau maximum principle holds on $\Sigma$ for the Laplacian operator, so that we may apply Lemma 14. Thus, in the case where $\Theta \leq 0$, we have $\mathcal{H}(h) \geq 0$ and therefore

$$
(-1)^{j} \mathcal{H}(h)^{k-1-j} \Theta^{j} \geq 0
$$

for every $j=0, \ldots, k-1$. Since the operators $P_{0}=I, P_{1}, \ldots, P_{k-1}$ are all positive definite, it follows from here that the operator $\mathcal{P}_{k-1}$ is positive semi-definite or, in other words, that the differential operator $\mathcal{L}_{k-1}$ is semi-elliptic. Furthermore, since $0 \leq-\Theta \leq 1$,

$$
\operatorname{Tr}\left(\mathcal{P}_{k-1}\right)=c_{k-1} \sum_{j=0}^{k-1}(-1)^{j} \mathcal{H}(h)^{k-1-j} \Theta^{j} H_{j} \leq c_{k-1} \sum_{j=0}^{k-1} \mathcal{H}\left(h^{*}\right)^{k-1-j} H_{j}^{*},
$$

where $h^{*}=\sup _{\Sigma} h<+\infty$ and $H_{j}^{*}=\sup _{\Sigma} H_{j} \leq\left(\sup _{\Sigma} H_{1}\right)^{j}<+\infty$ because of (1). Hence by Corollary 3 , the Omori-Yau maximum principle holds for the operator $\mathcal{L}_{k-1}$ and, proceeding as in the proof of Theorem 16, we may find two sequences $\left\{p_{j}\right\}_{j \in \mathbb{N}} \subset \Sigma$ and $\left\{q_{j}\right\}_{j \in \mathbb{N}} \subset \Sigma$ satisfying

$$
\begin{gathered}
\lim _{j \rightarrow+\infty} h\left(p_{j}\right)=h^{*}, \quad \text { and } \lim _{j \rightarrow+\infty} h\left(q_{j}\right)=h_{*}, \\
\lim _{j \rightarrow+\infty} \Theta\left(p_{j}\right)=\lim _{j \rightarrow+\infty} \Theta\left(q_{j}\right)=-1, \\
\mathcal{L}_{k-1} \sigma(h)\left(p_{j}\right)=c_{k-1} \rho\left(h\left(p_{j}\right)\right)\left(\mathcal{H}\left(h\left(p_{j}\right)\right)^{k}+(-1)^{k-1} \Theta^{k}\left(p_{j}\right) H_{k}\right)<\frac{1}{j},
\end{gathered}
$$

and

$$
\mathcal{L}_{k-1} \sigma(h)\left(q_{j}\right)=c_{k-1} \rho\left(h\left(q_{j}\right)\right)\left(\mathcal{H}\left(h\left(q_{j}\right)\right)^{k}+(-1)^{k-1} \Theta^{k}\left(q_{j}\right) H_{k}\right)<\frac{1}{j} .
$$

Making $j \rightarrow+\infty$ in the inequalties above, we obtain that

$$
\mathcal{H}\left(h^{*}\right)^{k} \leq H_{k} \leq \mathcal{H}\left(h_{*}\right)^{k},
$$

which implies that $h_{*}=h^{*}$, as in the proof of Theorem 16 . 
Finally, in the case where $\Theta \geq 0$ we proceed again as in the proof of Theorem 16. working now with the operator $(-1)^{k-1} \mathcal{L}_{k-1}$, which in this case is semi-elliptic and with $\operatorname{Tr}\left((-1)^{k-1} \mathcal{P}_{k-1}\right)$ bounded from above.

As in the previous section for Theorem 19, Theorem 21 remains true if we replace condition (30) by the stronger condition of $\Sigma^{n}$ having radial sectional curvature bounded from below by a constant. By applying Lemma 18, we see that this happens when the sectional curvature of $\mathbb{P}^{n}$ is itself bounded from below.

Corollary 22. Let $\mathbb{P}^{n}$ be a complete Riemannian manifold with sectional curvature bounded from below, and let $f: \Sigma^{n} \rightarrow I \times{ }_{\rho} \mathbb{P}^{n}$ be a complete hypersurface with constant $k$-mean curvature $H_{k}, 3 \leq k \leq n$. Assume that there exists an elliptic point in $\Sigma, \sup _{\Sigma}\left|H_{1}\right|<+\infty$ and $\Sigma$ is contained in a slab. If $\mathcal{H}^{\prime}(t)>0$ almost everywhere and the angle function $\Theta$ does not change sign, then $f\left(\Sigma^{n}\right)$ is a slice.

Indeed, by (11) we know that $H_{2}>0$, so that $\sup _{\Sigma}\|A\|^{2} \leq n^{2}\left(\sup _{\Sigma} H_{1}\right)^{2}<+\infty$ and we may apply Lemma 14 to conclude that the radial sectional curvature of $\Sigma$ is bounded from below. The result then follows from Theorem 21.

Finally, similar to what happened in the previous section, condition (30) has been used in the proof of Theorem 21 only to guarantee that the Omori-Yau maximum principle holds on $\Sigma$ for the Laplacian and for the semi-elliptic operator $\mathcal{L}_{k-1}$ (or $-\mathcal{L}_{k-1}$ ). Therefore, the theorem remains true under any other hypothesis guaranteeing that property. Then, and as a consequence of Corollary 4, we can also state the following:

Theorem 23. Let $\mathbb{P}^{n}$ be a complete, non-compact, Riemannian manifold whose radial sectional curvature satisfies condition (13). Let $f: \Sigma^{n} \rightarrow I \times{ }_{\rho} \mathbb{P}^{n}$ be a properly immersed hypersurface of constant $k$-mean curvature, $3 \leq k \leq n$. Assume that there exists an elliptic point in $\Sigma, \sup _{\Sigma}\left|H_{1}\right|<+\infty$ and that $\Sigma$ is contained in a slab. If $\mathcal{H}^{\prime}(t)>0$ almost everywhere and the angle function $\Theta$ does not change sign, then $f\left(\Sigma^{n}\right)$ is a slice.

\section{FURTHER RESULTS FOR HYPERSURFACES WITH CONSTANT HIGHER ORDER MEAN CURVATURES}

In this last section we introduce some further results for the case of constant higher order mean curvatures. As a first result in this direction we shall prove the next

Theorem 24. Let $f: \Sigma^{n} \rightarrow M^{n+1}=I \times{ }_{\rho} \mathbb{P}^{n}$ be a compact hypersurface of constant $k$-mean curvature, $2 \leq k \leq n$, and suppose that $\mathcal{H}$ does not vanish. Assume that

$$
K_{\mathbb{P} n} \geq \sup _{I}\left\{\rho^{\prime 2}-\rho^{\prime \prime} \rho\right\}
$$

$K_{\mathbb{P} n}$ being the sectional curvature of $\mathbb{P}^{n}$, and that the angle function $\Theta$ does not change sign. Then either $f\left(\Sigma^{n}\right)$ is a slice over a compact $\mathbb{P}^{n}$ or $M^{n+1}$ has constant sectional curvature and $\Sigma^{n}$ is a geodesic hypersphere. The latter case cannot occur if the inequality (35) is strict.

First we proceed with the proof of two important lemmas that will be essential in the proof of Theorem 24. For Lemma 25] see also Lemma 3.1 in [5]. 
Lemma 25. Let $\Sigma^{n} \rightarrow \bar{M}^{n+1}$ be an isometric immersion. Let $E_{1}, \ldots, E_{n}$ be a local orthonormal frame on $\Sigma$, and let $N$ be local unit normal. Then

$$
\sum_{i=1}^{n}\left\langle\left(\nabla_{E_{i}} P_{k}\right) X, E_{i}\right\rangle=\sum_{j=0}^{k-1} \sum_{i=1}^{n}(-1)^{k-1-j}\left\langle\overline{\mathrm{R}}\left(E_{i}, A^{k-1-j} X\right) N, P_{j} E_{i}\right\rangle,
$$

for every vector field $X \in T \Sigma$.

Proof. We will prove equation (36) by induction on $k, 1 \leq k \leq n-1$. It is not difficult to prove that this is true for $k=1$ using the Codazzi equation and the definition of $P_{1}$. Assume that the equation holds for $k-1$. Then, again using the Codazzi equation, we get

$$
\begin{aligned}
\sum_{i=1}^{n}\left\langle\left(\nabla_{E_{i}} P_{k}\right) X, E_{i}\right\rangle= & -\sum_{i=1}^{n}\left\langle\left(\nabla_{E_{i}} P_{k-1}\right) A X, E_{i}\right\rangle+\sum_{i=1}^{n}\left\langle\overline{\mathrm{R}}\left(E_{i}, X\right) N, P_{k-1} E_{i}\right\rangle \\
= & -\sum_{j=0}^{k-2} \sum_{i=1}^{n}(-1)^{k-2-j}\left\langle\overline{\mathrm{R}}\left(E_{i}, A^{k-1-j} X\right) N, P_{j} E_{i}\right\rangle \\
& +\sum_{i=1}^{n}\left\langle\overline{\mathrm{R}}\left(E_{i}, X\right) N, P_{k-1} E_{i}\right\rangle \\
= & \sum_{j=0}^{k-1} \sum_{i=1}^{n}(-1)^{k-1-j}\left\langle\overline{\mathrm{R}}\left(E_{i}, A^{k-1-j} X\right) N, P_{j} E_{i}\right\rangle .
\end{aligned}
$$

For further details see [5], paying attention to the different conventions for the sign of $\overline{\mathrm{R}}$.

Corollary 26. Let $\Sigma^{n} \rightarrow I \times{ }_{\rho} \mathbb{P}^{n}$ be an immersed hypersurface, and assume that $\mathbb{P}^{n}$ has constant sectional curvature $\kappa$. Then

$$
\operatorname{div} P_{k}=-(n-k) \Theta\left(\frac{\kappa}{\rho^{2}(h)}+\mathcal{H}^{\prime}(h)\right) P_{k-1} \nabla h .
$$

Proof. Let $E_{1}, \ldots, E_{n}$ be a local orthonormal frame on $\Sigma^{n}$, and observe that

$$
\left\langle\operatorname{div} P_{k}, X\right\rangle=\sum_{i=1}^{n}\left\langle\left(\nabla_{E_{i}} P_{k}\right) X, E_{i}\right\rangle
$$

for every vector field $X \in T \Sigma$. Fix $j, j=0, \ldots, k-1$. Then

$$
\begin{aligned}
\sum_{i=1}^{n}\left\langle\overline{\mathrm{R}}\left(E_{i}, A^{k-1-j} X\right) N, P_{j} E_{i}\right\rangle= & \sum_{i=1}^{n}\left\langle\mathrm{R}_{\mathbb{P}}\left(\pi_{\mathbb{P}_{*}} E_{i}, \pi_{\mathbb{P}_{*}} A^{k-1-j} X\right) \pi_{\mathbb{P}_{*}} N, P_{j} E_{i}\right\rangle \\
& +\Theta \mathcal{H}^{\prime}(h)\left(\left\langle P_{j} \nabla h, A^{k-1-j} X\right\rangle\right. \\
& \left.-c_{j} H_{j}\left\langle\nabla h, A^{k-1-j} X\right\rangle\right) .
\end{aligned}
$$

Since $\mathbb{P}^{n}$ has constant sectional curvature $\kappa$ it follows that

$$
\mathrm{R}_{\mathbb{P}}(Y, Z) W=\kappa\left(\langle Z, W\rangle_{\mathbb{P}} Y-\langle Y, W\rangle_{\mathbb{P}} Z\right) .
$$

Hence a direct calculation shows that

$$
\begin{aligned}
\sum_{i=1}^{n}\left\langle R_{\mathbb{P}}\left(\pi_{\mathbb{P}_{*}} E_{i}, \pi_{\mathbb{P}_{*}} A^{k-1-j} X\right) \pi_{\mathbb{P}_{*}} N, P_{j} E_{i}\right\rangle= & \frac{\kappa}{\rho^{2}(h)} \Theta\left(\left\langle P_{j} \nabla h, A^{k-1-j} X\right\rangle\right. \\
& \left.-c_{j} H_{j}\left\langle\nabla h, A^{k-1-j} X\right\rangle\right) .
\end{aligned}
$$


We claim that

$$
B_{k}:=\sum_{j=0}^{k-1}(-1)^{k-j-1}\left(P_{j} A^{k-1-j}-c_{j} H_{j} A^{k-1-j}\right)=-(n-k) P_{k-1} .
$$

In this case the conclusion of Corollary 26 is immediate. We prove the claim by induction on $k, k=0, \ldots, n-1$. The case $k=0$ is trivial. Assume that we proved the equation for $k-1$. Then

$$
\begin{aligned}
B_{k} & =P_{k-1}-c_{k-1} H_{k-1} I-B_{k-1} \circ A \\
& =P_{k-1}-c_{k-1} H_{k-1} I+(n-k+1) P_{k-2} A \\
& =-(n-k) P_{k-1} .
\end{aligned}
$$

Lemma 27. Let $\Sigma^{n}$ be a hypersurface immersed into a warped product space $I \times \mathbb{P}^{n}$, with angle function $\Theta$ and height function $h$. Let $\hat{\Theta}=\rho \Theta$. Then, for every $k=0, \ldots, n-1$, we have

$$
\begin{aligned}
L_{k} \hat{\Theta}= & -\left(\begin{array}{c}
n \\
k+1
\end{array}\right) \rho(h)\left\langle\nabla h, \nabla H_{k+1}\right\rangle-\rho^{\prime}(h) c_{k} H_{k+1} \\
& -\hat{\Theta} \mathcal{H}^{\prime}(h)\left(\|\nabla h\|^{2} c_{k} H_{k}-\left\langle P_{k} \nabla h, \nabla h\right\rangle\right)-\frac{\hat{\Theta}}{\rho(h)^{2}} \beta_{k} \\
& -\hat{\Theta}\left(\begin{array}{c}
n \\
k+1
\end{array}\right)\left(n H_{1} H_{k+1}-(n-k-1) H_{k+2}\right),
\end{aligned}
$$

where

$$
\beta_{k}=\sum_{i=1}^{n} \mu_{k, i} K_{\mathbb{P}}\left(\pi_{\mathbb{P} *} E_{i}, \pi_{\mathbb{P} *} N\right)\left\|\pi_{\mathbb{P} *} E_{i} \wedge \pi_{\mathbb{P} *} N\right\|^{2} .
$$

Here the $\mu_{k, i}$ 's stand for the eigenvalues of $P_{k}$ and $\left\{E_{1}, \ldots, E_{n}\right\}$ is a local orthonormal frame on $\Sigma$ diagonalizing $A$.

Proof. Since $\rho(t) T$ is a conformal vector field

$$
\nabla \hat{\Theta}=-\rho(h) A \nabla h .
$$

Therefore, using equation (24) we find

$$
\nabla_{X} \nabla \hat{\Theta}=-\rho(h)\left(\nabla_{X} A\right) \nabla h-\rho^{\prime}(h) A X-\hat{\Theta} A^{2} X .
$$

Hence

$$
\begin{aligned}
L_{k} \hat{\Theta}= & -\rho(h) \sum_{i=1}^{n}\left\langle P_{k}\left(\nabla_{E_{i}} A\right) \nabla h, E_{i}\right\rangle \\
& -\rho^{\prime}(h) c_{k} H_{k+1}-\left(\begin{array}{c}
n \\
k+1
\end{array}\right) \hat{\Theta}\left(H_{1} H_{k+1}-(n-k-1) H_{k+2}\right) .
\end{aligned}
$$

Using the expression of the covariant derivative of a tensor field. we get

$$
\begin{aligned}
-P_{k}\left(\nabla_{E_{i}} A\right) \nabla h & =\left(\nabla_{E_{i}} P_{k}\right) A \nabla h-\left(\nabla_{E_{i}} P_{k} A\right) \nabla h \\
& =\left(\nabla_{E_{i}} P_{k}\right) A \nabla h+\left(\nabla_{E_{i}} P_{k+1}\right) \nabla h-E_{i}\left(S_{k+1}\right) \nabla h .
\end{aligned}
$$


By equation (36) it follows that

$$
\begin{aligned}
-\sum_{i=1}^{n}\left\langle P_{k}\left(\nabla_{E_{i}} A\right) \nabla h, E_{i}\right\rangle= & \sum_{i=1}^{n}\left\langle\left(\nabla_{E_{i}} P_{k}\right) A \nabla h, E_{i}\right\rangle \\
& +\sum_{i=1}^{n}\left\langle\left(\nabla_{E_{i}} P_{k+1}\right) \nabla h, E_{i}\right\rangle-\nabla h\left(S_{k+1}\right) \\
= & \sum_{i=1}^{n}\left\langle\overline{\mathrm{R}}\left(E_{i}, \nabla h\right) N, P_{k} E_{i}\right\rangle-\nabla h\left(S_{k+1}\right) .
\end{aligned}
$$

Since $\nabla h=T-\Theta N$, we can write

$$
\overline{\mathrm{R}}\left(E_{i}, \nabla h\right) N=\overline{\mathrm{R}}\left(E_{i}, T\right) N-\Theta \overline{\mathrm{R}}\left(E_{i}, N\right) N .
$$

Using the Gauss equation and observing that $\pi_{\mathbb{P}_{*}} T=0$, we get

$$
\overline{\mathrm{R}}\left(E_{i}, T\right) N=-\left(\mathcal{H}(h)^{2}+\mathcal{H}^{\prime}(h)\right) \Theta E_{i}=-\frac{\rho^{\prime \prime}(h)}{\rho(h)} \Theta E_{i} .
$$

So

$$
\sum_{i=1}^{n}\left\langle\overline{\mathrm{R}}\left(E_{i}, T\right) N, P_{k} E_{i}\right\rangle=-\frac{\rho^{\prime \prime}(h)}{\rho(h)} \Theta c_{k} H_{k} .
$$

Again by the Gauss equation

$$
\begin{aligned}
\overline{\mathrm{R}}\left(E_{i}, N\right) N= & \mathrm{R}_{\mathbb{P}}\left(\pi_{\mathbb{P}_{*}} E_{i}, \pi_{\mathbb{P}_{*}} N\right) \pi_{\mathbb{P} *} N-\mathcal{H}(h)^{2} E_{i} \\
& +\mathcal{H}^{\prime}(h) \Theta\left(\left\langle E_{i}, \nabla h\right\rangle N-\Theta E_{i}\right)-\mathcal{H}^{\prime}(h)\left\langle E_{i}, \nabla h\right\rangle T .
\end{aligned}
$$

Assume that the orthonormal basis $\left\{E_{i}\right\}_{1}^{n}$ diagonalizes $A$ and hence $P_{k}$, that is $P_{k} E_{i}=\mu_{k, i} E_{i}$. Then

$$
\begin{aligned}
\sum_{i=1}^{n}\left\langle\overline{\mathrm{R}}\left(E_{i}, N\right) N, P_{k} E_{i}\right\rangle= & \frac{1}{\rho(h)^{2}} \sum_{i=1}^{n} \mu_{k, i} K_{\mathbb{P}}\left(\pi_{\mathbb{P}_{*}} E_{i}, \pi_{\mathbb{P}_{*}} N\right)\left\|\pi_{\mathbb{P}_{*}} E_{i} \wedge \pi_{\mathbb{P}_{*}} N\right\|^{2} \\
& -\frac{\rho^{\prime \prime}(h)}{\rho(h)} c_{k} H_{k}+\mathcal{H}^{\prime}(h)\left(\|\nabla h\|^{2} c_{k} H_{k}-\left\langle P_{k} \nabla h, \nabla h\right\rangle\right) .
\end{aligned}
$$

Thus,

$$
\begin{aligned}
\sum_{i=1}^{n}\left\langle\overline{\mathrm{R}}\left(E_{i}, \nabla h\right) N, P_{k} E_{i}\right\rangle & =\sum_{i=1}^{n}\left\langle\overline{\mathrm{R}}\left(E_{i}, T\right) N, P_{k} E_{i}\right\rangle-\Theta \sum_{i=1}^{n}\left\langle\overline{\mathrm{R}}\left(E_{i}, N\right) N, P_{k} E_{i}\right\rangle \\
& =-\frac{\Theta}{\rho(h)^{2}} \sum_{i=1}^{n} \mu_{k, i} K_{\mathbb{P}}\left(\pi_{\mathbb{P}_{*}} E_{i}, \pi_{\mathbb{P} *} N\right)\left\|\pi_{\mathbb{P}_{*}} E_{i} \wedge \pi_{\mathbb{P} *} N\right\|^{2} \\
& -\Theta \mathcal{H}^{\prime}(h)\left(\|\nabla h\|^{2} c_{k} H_{k}-\left\langle P_{k} \nabla h, \nabla h\right\rangle\right),
\end{aligned}
$$

and this concludes the proof of the lemma.

Corollary 28. Let $\Sigma^{n}$ be a hypersurface immersed into a warped product space $I \times{ }_{\rho} \mathbb{P}^{n}$ with angle function $\Theta$ and height function $h$. Assume that $\mathbb{P}^{n}$ has constant 
sectional curvature $\kappa$, and let $\hat{\Theta}=\rho(h) \Theta$. Then, for every $k=0, \ldots, n-1$ we have

$$
\begin{aligned}
L_{k} \hat{\Theta}= & -\left(\begin{array}{c}
n \\
k+1
\end{array}\right) \rho(h)\left\langle\nabla h, \nabla H_{k+1}\right\rangle-\rho^{\prime}(h) c_{k} H_{k+1} \\
& -\hat{\Theta}\left(\frac{\kappa}{\rho^{2}(h)}+\mathcal{H}^{\prime}(h)\right)\left(\|\nabla h\|^{2} c_{k} H_{k}-\left\langle P_{k} \nabla h, \nabla h\right\rangle\right) \\
& -\hat{\Theta}\left(\begin{array}{c}
n \\
k+1
\end{array}\right)\left(n H_{1} H_{k+1}-(n-k-1) H_{k+2}\right) .
\end{aligned}
$$

We are now ready to give the

Proof of Theorem 24. We may assume without loss of generality that $\mathcal{H}(h)>0$ on $\Sigma$. Since $\Sigma^{n}$ is compact, there exists a point $p_{0} \in \Sigma$ where the height function attains its maximum. Then $\nabla h\left(p_{0}\right)=0, \Theta\left(p_{0}\right)= \pm 1$ and by (24)

$$
\operatorname{Hess} h\left(p_{0}\right)(v, v)=\mathcal{H}\left(h^{*}\right)\langle v, v\rangle+\Theta\left(p_{0}\right)\langle A v, v\rangle\left(p_{0}\right) \leq 0 .
$$

If $\Theta\left(p_{0}\right)=-1$, then

$$
\langle A v, v\rangle\left(p_{0}\right) \geq \mathcal{H}\left(h^{*}\right)\langle v, v\rangle>0
$$

for any $v \neq 0$. Thus $p_{0}$ is an elliptic point, $H_{k}$ is a positive constant and by the Gårding inequalities

$$
H_{1} \geq H_{2}^{\frac{1}{2}} \geq \cdots \geq H_{k}^{\frac{1}{k}}>0
$$

with equality only at umbilical points. In particular, $\Sigma$ is two sided and then $\Theta \leq 0$. If $\Theta\left(p_{0}\right)=1$, changing the orientation we have the same conclusion.

Consider the function

$$
\phi=\sigma(h) H_{k}^{\frac{1}{k}}+\rho(h) \Theta .
$$

Let us prove that $L_{k-1} \phi \geq 0$. Since $H_{k}$ is constant, we have

$$
\begin{aligned}
L_{k-1} \phi= & H_{k}^{\frac{1}{k}} L_{k-1} \sigma(h)+L_{k-1} \hat{\Theta} \\
= & c_{k-1} H_{k}^{\frac{1}{k}}\left(\rho^{\prime}(h) H_{k-1}+\hat{\Theta} H_{k}\right)-c_{k-1} H_{k-1} \hat{\Theta} \mathcal{H}^{\prime}(h)\|\nabla h\|^{2} \\
& +\hat{\Theta} \mathcal{H}^{\prime}(h)\left\langle P_{k-1} \nabla h, \nabla h\right\rangle-\hat{\Theta}\left(\begin{array}{c}
n \\
k
\end{array}\right)\left(n H_{1} H_{k}-(n-k) H_{k+1}\right) \\
& -\rho^{\prime}(h) c_{k-1} H_{k}-\frac{\hat{\Theta}}{\rho(h)^{2}} \sum_{i=1}^{n} \mu_{k-1, i} K_{\mathbb{P}}\left(\pi_{\mathbb{P}_{*}} E_{i}, \pi_{\mathbb{P}_{*}} N\right)\left\|\pi_{\mathbb{P}_{*}} E_{i} \wedge \pi_{\mathbb{P}_{*}} N\right\|^{2} \\
= & A+B+C,
\end{aligned}
$$

where

$$
\begin{gathered}
A=-\hat{\Theta}\left(\begin{array}{l}
n \\
k
\end{array}\right)\left(n H_{1} H_{k}-(n-k) H_{k+1}-k H_{k}^{\frac{k+1}{k}}\right), \\
B=c_{k-1} \rho^{\prime}(h)\left(H_{k-1} H_{k}^{\frac{1}{k}}-H_{k}\right)
\end{gathered}
$$

and

$$
\begin{aligned}
C= & -\hat{\Theta} \mathcal{H}^{\prime}(h)\left(\|\nabla h\|^{2} c_{k-1} H_{k-1}-\left\langle P_{k-1} \nabla h, \nabla h\right\rangle\right) \\
& -\frac{\hat{\Theta}}{\rho(h)^{2}} \sum_{i=1}^{n} \mu_{k-1, i} K_{\mathbb{P}}\left(\pi_{\mathbb{P}_{*}} E_{i}, \pi_{\mathbb{P}_{*}} N\right)\left\|\pi_{\mathbb{P}_{*}} E_{i} \wedge \pi_{\mathbb{P}_{*}} N\right\|^{2} .
\end{aligned}
$$

Then by the Gårding inequalities,

$$
H_{k-1} H_{k}^{\frac{1}{k}}-H_{k}=H_{k}^{\frac{1}{k}}\left(H_{k-1}-H_{k}^{\frac{k-1}{k}}\right) \geq 0 .
$$


Moreover,

hence

$$
n H_{1} H_{k}-k H_{k}^{\frac{k+1}{k}} \geq n H_{k}^{\frac{k+1}{k}}-k H_{k}^{\frac{k+1}{k}}=(n-k) H_{k}^{\frac{k+1}{k}}
$$

$$
n H_{1} H_{k}-k H_{k}^{\frac{k+1}{k}}-(n-k) H_{k+1} \geq(n-k)\left(H_{k}^{\frac{k+1}{k}}-H_{k+1}\right) \geq 0 .
$$

Finally, let $\alpha:=\sup _{I}\left\{\rho^{\prime 2}-\rho^{\prime \prime} \rho\right\}$. Since

$$
\left\|\pi_{\mathbb{P}_{*}} E_{i} \wedge \pi_{\mathbb{P}_{*}} N\right\|^{2}=\|\nabla h\|^{2}-\left\langle E_{i}, \nabla h\right\rangle^{2},
$$

taking into account that the $\mu_{k-1, i}$ 's are positive, we have

$$
\begin{aligned}
& \sum_{i=1}^{n} \mu_{k-1, i} K_{\mathbb{P}}\left(\pi_{\mathbb{P}_{*}} E_{i}, \pi_{\mathbb{P}_{*}} N\right)\left\|\pi_{\mathbb{P}_{*}} E_{i} \wedge \pi_{\mathbb{P}_{*}} N\right\|^{2} \\
& \geq \alpha \sum_{i=1}^{n} \mu_{k-1, i}\left\|\pi_{\mathbb{P}_{*}} E_{i} \wedge \pi_{\mathbb{P}_{*}} N\right\|^{2} \\
& =\alpha\left(c_{k-1} H_{k-1}\|\nabla h\|^{2}-\left\langle P_{k-1} \nabla h, \nabla h\right\rangle\right) .
\end{aligned}
$$

Hence,

$$
\begin{aligned}
\frac{1}{\rho(h)^{2}} & \sum_{i=1}^{n} \mu_{k-1, i} K_{\mathbb{P}}\left(\pi_{\mathbb{P}_{*}} E_{i}, \pi_{\mathbb{P}_{*}} N\right)\left\|\pi_{\mathbb{P}_{*}} E_{i} \wedge \pi_{\mathbb{P}_{*}} N\right\|^{2} \\
& +\mathcal{H}^{\prime}(h)\left(\|\nabla h\|^{2} c_{k-1} H_{k-1}-\left\langle P_{k-1} \nabla h, \nabla h\right\rangle\right) \\
\geq & \left(\frac{\alpha}{\rho(h)^{2}}+\mathcal{H}^{\prime}(h)\right)\left(\|\nabla h\|^{2} c_{k-1} H_{k-1}-\left\langle P_{k-1} \nabla h, \nabla h\right\rangle\right) \geq 0,
\end{aligned}
$$

where the last inequality follows from $\alpha=\sup _{I}\left\{-\rho^{2} \mathcal{H}^{\prime}\right\}$ and from the fact that $P_{k-1}$ is a positive definite operator. Thus, $L_{k-1} \phi \geq 0$. Since $L_{k-1}$ is an elliptic operator and $\Sigma$ is compact, we conclude by the maximum principle that $\phi$ must be constant. Hence $L_{k-1} \phi=0$ and the three terms $A, B$, and $C$ in $L_{k-1} \phi$ vanish on $\Sigma$.

In particular $B=0$ implies that $\Sigma$ is a totally umbilical hypersurface. Moreover, since $H_{k}$ is a positive constant and $\Sigma$ is totally umbilical, all the higher order mean curvatures are constant. In particular, $H_{1}$ is constant and the conclusion follows by Theorem 3.4 [4.

On the other hand, using Theorem 3.1 in 4 we can give the following version of Theorem 3.4 in [4] for the complete case.

Proposition 29. Let $M^{n+1}=I \times{ }_{\rho} \mathbb{P}^{n}$ be a warped product space, and assume that the Ricci curvature of $\mathbb{P}^{n}$ satisfies

$$
\operatorname{Ric}_{\mathbb{P}}>\sup _{I}\left\{\rho^{\prime 2}-\rho^{\prime \prime} \rho\right\} .
$$

Let $f: \Sigma^{n} \rightarrow I \times{ }_{\rho} \mathbb{P}^{n}$ be a complete, parabolic, two sided hypersurface with constant mean curvature. Suppose that

$$
f\left(\Sigma^{n}\right) \subset\left[t_{1}, t_{2}\right] \times \mathbb{P}^{n},
$$

where $t_{1}, t_{2}$ are finite. If the angle function $\Theta$ does not change sign, then $f\left(\Sigma^{n}\right)$ is a slice. 
For the proof, observe that since the function $\phi=H \sigma(h)+\hat{\Theta}$ is subharmonic and $\Sigma$ is parabolic, then it must be constant. In particular, $\Delta \phi=0$ and by equation (3.8) in 4] we conclude that $h$ has to be constant, because of the strict inequality in (38).

In what follows, we extend this result to higher order mean curvatures. Towards this aim, we let $\mathfrak{L}_{k}$ be the operator

$$
\mathfrak{L}_{k} f=\operatorname{div}\left(P_{k} \nabla f\right),
$$

where $f \in C^{\infty}(\Sigma)$. Notice that

$$
\mathfrak{L}_{k} f=\left\langle\operatorname{div} P_{k}, \nabla f\right\rangle+L_{k} f .
$$

We introduce the following

Definition 30. We will say that the manifold $\Sigma^{n} \hookrightarrow I \times{ }_{\rho} \mathbb{P}^{n}$ is $\mathfrak{L}_{k}$-parabolic if the only bounded above $C^{1}$ solutions of the inequality

$$
\mathfrak{L}_{k} f \geq 0
$$

are constant.

The following theorem is a special case of Theorem 2.6 in [13].

Theorem 31. Let $\Sigma^{n} \hookrightarrow I \times{ }_{\rho} \mathbb{P}^{n}$ be a complete manifold. Fix an origin $o \in \Sigma$. If

$$
\left(\sup _{\partial B_{t}} H_{k-1} \operatorname{vol}\left(\partial B_{t}\right)\right)^{-1} \notin L^{1}(+\infty),
$$

where $\partial B_{t}$ is the geodesic sphere of radius t centered at o, then $\Sigma^{n}$ is $\mathfrak{L}_{k-1}$-parabolic.

We are ready to state our last result.

Theorem 32. Let $M^{n+1}=I \times{ }_{\rho} \mathbb{P}^{n}$ be a warped product space, and assume that $\mathbb{P}^{n}$ has constant sectional curvature $\kappa$ satisfying

$$
\kappa>\sup _{I}\left\{\rho^{\prime 2}-\rho^{\prime \prime} \rho\right\}
$$

Let $f: \Sigma^{n} \rightarrow I \times_{\rho} \mathbb{P}^{n}$ be a complete hypersurface with $\sup _{\Sigma}\left|H_{1}\right|<+\infty$ and satisfying condition (39). Suppose that $f$ has constant $k$-mean curvature, $2 \leq k \leq$ $n$, and

$$
f\left(\Sigma^{n}\right) \subset\left[t_{1}, t_{2}\right] \times \mathbb{P}^{n},
$$

where $t_{1}, t_{2}$ are finite. Assume that either $k=2$ and $H_{2}$ is positive or $k \geq 3$ and there exists an elliptic point $p \in \Sigma^{n}$. If $\mathcal{H}(h)$ and the angle function $\Theta$ do not change sign, then $f\left(\Sigma^{n}\right)$ is a slice.

Remark 33. Comparing with Theorem 24, we have relaxed the condition on $\mathcal{H}$ but we are requiring, as it will be clear from the proof, the existence of an elliptic point. That, on a compact manifold was guaranteed by the assumption $\mathcal{H}>0$. Moreover, we observe that the angle function is indeed well defined because $\Sigma$ is two sided. For $k=2$, this follows from the positivity of $H_{2}$ since $H_{1}^{2} \geq H_{2}>0$. In the remaining cases this property follows from the Gårding inequalities, as in the compact case. In any case we choose the orientation so that $H_{1}>0$.

Proof. It follows from the hypotheses that $\sup _{\Sigma}\|A\|<+\infty$ and therefore by Lemma 18 that the sectional curvature of $\Sigma$ is bounded from below. We then deduce the validity of the Omori-Yau maximum principle for the Laplacian. Assume $\mathcal{H}(h) \geq 0$. 
Applying the Omori-Yau maximum principle to the Laplace operator and using equation (21) we find that

$$
-\operatorname{sgn} \Theta \liminf _{j \rightarrow+\infty} H_{1}\left(q_{j}\right) \geq \mathcal{H}\left(h^{*}\right) \geq 0 .
$$

Therefore for the chosen orientation, $\operatorname{sgn} \Theta=-1$ and $\Theta \leq 0$ on $\Sigma$. Consider the operator

$$
\mathfrak{L}_{k-1} f=\operatorname{div}\left(P_{k-1} \nabla f\right)
$$

and the function

$$
\phi=H_{k}^{\frac{1}{k}} \sigma(h)+\hat{\Theta},
$$

where $\hat{\Theta}=\rho(h) \Theta$. Since $\mathbb{P}^{n}$ has constant sectional curvature $\kappa$, it follows by equation (37) that

$$
\begin{aligned}
\mathfrak{L}_{k-1} \phi= & -(n-k+1) \Theta\left(\frac{\kappa}{\rho^{2}(h)}+\mathcal{H}^{\prime}(h)\right)\left\langle P_{k-2} \nabla h, \nabla \phi\right\rangle+L_{k-1} \phi \\
= & -(n-k+1) \hat{\Theta}\left(\frac{\kappa}{\rho^{2}(h)}+\mathcal{H}^{\prime}(h)\right)\left\langle P_{k-2} \nabla h, \nabla h\right\rangle H_{k}^{\frac{1}{k}} \\
& +(n-k+1) \hat{\Theta}\left(\frac{\kappa}{\rho^{2}(h)}+\mathcal{H}^{\prime}(h)\right)\left\langle P_{k-2} A \nabla h, \nabla h\right\rangle \\
& +H_{k}^{\frac{1}{k}} L_{k-1} \sigma(h)+L_{k-1} \hat{\Theta} .
\end{aligned}
$$

Using equation (22) and Corollary 28, we find

$$
\begin{aligned}
\mathfrak{L}_{k-1} \phi= & c_{k-1} \rho^{\prime}(h) H_{k}^{\frac{1}{k}}\left(H_{k-1}-H_{k}^{\frac{k-1}{k}}\right) \\
& -\left(\begin{array}{c}
n \\
k
\end{array}\right) \hat{\Theta}\left(n H_{1} H_{k}-(n-k) H_{k+1}-k H_{k}^{\frac{k+1}{k}}\right) \\
& -(n-k) \hat{\Theta}\left(\frac{\kappa}{\rho^{2}(h)}+\mathcal{H}^{\prime}(h)\right)\left\langle P_{k-1} \nabla h, \nabla h\right\rangle \\
& -(n-k+1) \hat{\Theta} H_{k}^{\frac{1}{k}}\left(\frac{\kappa}{\rho^{2}(h)}+\mathcal{H}^{\prime}(h)\right)\left\langle P_{k-2} \nabla h, \nabla h\right\rangle .
\end{aligned}
$$

Using Gårding inequalities as in Theorem 24, it is easy to prove that the first and the second terms are nonnegative. By the fact that each $P_{j}$ is an elliptic operator, $j=0, \ldots, k-1$, and by equation (35), it follows that also all the remaining terms in the previous equation are nonnegative. Thus $\mathfrak{L}_{k-1} \phi \geq 0$. Since, by assumption (39) $\Sigma^{n}$ is $\mathfrak{L}_{k-1}$-parabolic, we conclude that $\phi$ has to be constant. In particular, $\mathfrak{L}_{k-1} \phi=0$ and the four terms on the right-hand side of equation (41) vanish. Let us prove that $\mathcal{U}=\left\{p \in \Sigma^{n}: \Theta(p)=0\right\}$ has empty interior. Indeed, assume the contrary, and let $\mathcal{V} \neq \emptyset$ be an open subset of $\mathcal{U}$. On $\mathcal{V}$ the function $\phi=\sigma(h) H_{k}^{1 / k}$ is constant. Hence, since $H_{k} \neq 0$, then $\sigma(h)$ and, equivalently $h$, is constant on $\mathcal{V}$, which is not possible since $\|\nabla h\|^{2}=1-\Theta^{2}=1$ on $\mathcal{V}$. Therefore, since the third term on the right-hand of (41) vanishes identically, we have

$$
\left\langle P_{k-1} \nabla h, \nabla h\right\rangle=0 .
$$

Since $P_{k-1}$ is positive definite, this means that $h$ has to be constant. 


\section{REFERENCES}

1. A. D. Alexandrov, A characteristic property of spheres, Ann. Mat. Pura Appl. (4) 58 (1962), 303-315. MR0143162(26:722)

2. L. J. Alías and A. G. Colares, Uniqueness of spacelike hypersurfaces with constant higher order mean curvature in generalized Robertson-Walker spacetimes, Math. Proc. Cambridge Philos. Soc. 143 (2007), no. 3, 703-729. MR2373968 (2008m:53146)

3. L. J. Alías and M. Dajczer, Uniqueness of constant mean curvature surfaces properly immersed in a slab, Comment. Math. Helv. 81 (2006), no. 3, 653-663. MR2250858(2007e:53003)

4. L. J. Alías and M. Dajczer, Constant mean curvature hypersurfaces in warped product spaces, Proc. Edinb. Math. Soc. (2) 50 (2007), 511-526. MR2360513 (2008k:53122)

5. L. J. Alías, J. H. S. de Lira, and J. M. Malacarne, Constant higher-order mean curvature hypersurfaces in Riemannian spaces, J. Inst. Math. Jussieu 5 (2006), no. 4, 527-562. MR2261223 (2007i:53062)

6. L. J. Alías, D. Impera, and M. Rigoli, Spacelike hypersurfaces of constant $k$-mean curvature in generalized Robertson-Walker spacetimes, Math. Proc. Cambridge Philos. Soc. 152 (2012), 365-383. MR2887879

7. J. L. M. Barbosa and A. G. Colares, Stability of Hypersurfaces with Constant r-Mean Curvature, Ann. Glob. An. Geom. 15 (1997), 277-297. MR1456513 (98h:53091)

8. M. F. Elbert, Constant positive 2-mean curvature hypersurfaces, Illinois J. Math. 46 (2002), no. 1, 247-267. MR.1936088 (2003g:53103)

9. L. Gårding, An inequality for hyperbolic polynomials, J. Math. Mech. 8 (1959), 957-965. MR0113978 (22:4809)

10. D. Gilbarg and N. S. Trudinger, Elliptic partial differential equations of second order, second ed., Grundlehren der Mathematischen Wissenschaften [Fundamental Principles of Mathematical Sciences], vol. 224, Springer-Verlag, Berlin, 1983. MR.737190 (86c:35035)

11. S. Montiel, Unicity of constant mean curvature hypersurfaces in some Riemannian manifolds, Indiana Univ. Math. J. 48 (1999), no. 2, 711-748. MR1722814 (2001f:53131)

12. H. Omori, Isometric immersions of Riemannian manifolds, J. Math. Soc. Japan 19 (1967), 205-214. MR0215259 (35:6101)

13. S. Pigola, M. Rigoli, and A. G. Setti, A Liouville-type result for quasi-linear elliptic equations on complete Riemannian manifolds, J. Funct. Anal. 219 (2005), no. 2, 400-432. MR.2109258 (2005j:35072)

14. Maximum principles on Riemannian manifolds and applications, Mem. Amer. Math. Soc. 174 (2005), no. 822, x+99. MR2116555(2006b:53048)

15. S. T. Yau, Harmonic functions on complete Riemannian manifolds, Comm. Pure Appl. Math. 28 (1975), 201-228. MR0431040 (55:4042)

Departamento de Matemáticas, Universidad de Murcia, Campus de Espinardo, 30100 EspinARdo, Murcia, SPAIN

E-mail address: ljalias@um.es

Dipartimento di Matematica, Università Degli studi di Milano, via Saldini 50, I-20133 Milano, ItAly

E-mail address: debora.impera@unimi.it

Dipartimento di Matematica, Università Degli Studi di Milano, via Saldini 50, I-20133 Milano, Italy

E-mail address: marco.rigoli@unimi.it 